\title{
Posterior thalamic nucleus axon terminals have different structure and functional impact in the motor and somatosensory vibrissal cortices
}

\author{
Diana Casas-Torremocha ${ }^{1}$. César Porrero ${ }^{1}$. Javier Rodriguez-Moreno ${ }^{1}$. María García-Amado ${ }^{1}$. \\ Joachim H. R. Lübke ${ }^{2,3,4}$ • Ángel Núñez ${ }^{1}$ · Francisco Clascá ${ }^{10}$
}

Received: 1 December 2018 / Accepted: 13 March 2019 / Published online: 27 March 2019

(c) The Author(s) 2019

\begin{abstract}
Rodents extract information about nearby objects from the movement of their whiskers through dynamic computations that are carried out by a network of forebrain structures that includes the thalamus and the primary sensory (S1BF) and motor (M1wk) whisker cortices. The posterior nucleus (Po), a higher order thalamic nucleus, is a key hub of this network, receiving cortical and brainstem sensory inputs and innervating both motor and sensory whisker-related cortical areas. In a recent study in rats, we showed that Po inputs differently impact sensory processing in S1BF and M1wk. Here, in C57BL/6 mice, we measured Po synaptic bouton layer distribution and size, compared cortical unit response latencies to "in vivo" Po activation, and pharmacologically examined the glutamatergic receptor mechanisms involved. We found that, in S1BF, a large majority (56\%) of Po axon varicosities are located in layer (L)5a and only 12\% in L2-L4, whereas in M1wk this proportion is inverted to $18 \%$ and 55\%, respectively. Light and electron microscopic measurements showed that Po synaptic boutons in M1wk layers 3-4 are significantly larger ( 50\%) than those in S1BF L5a. Electrical Po stimulation elicits different areaspecific response patterns. In S1BF, responses show weak or no facilitation, and involve both ionotropic and metabotropic glutamate receptors, whereas in M1wk, unit responses exhibit facilitation to repetitive stimulation and involve ionotropic NMDA glutamate receptors. Because of the different laminar distribution of axon terminals, synaptic bouton size and receptor mechanisms, the impact of Po signals on M1wk and S1BF, although simultaneous, is likely to be markedly different.
\end{abstract}

Keywords Cerebral cortex $\cdot$ Thalamocortical projections $\cdot$ Higher order thalamus $\cdot$ NMDA receptors $\cdot$ Metabotropic receptors $\cdot$ Excitatory synaptic boutons

Diana Casas-Torremocha and César Porrero share first authorship.

Electronic supplementary material The online version of this article (https://doi.org/10.1007/s00429-019-01862-4) contains supplementary material, which is available to authorized users.

Francisco Clascá

francisco.clasca@uam.es

1 Department of Anatomy and Graduate Program in Neuroscience, School of Medicine, Autónoma de Madrid University, Calle Arzobispo Morcillo 4, 28029 Madrid, Spain

2 Institute of Neuroscience and Medicine INM-10, Research Centre Jülich GmbH, 52425 Jülich, Germany

3 Department of Psychiatry, Psychotherapy and Psychosomatics, RWTH Aachen University, Aachen, Germany

4 JARA-Brain Medicine, Aachen, Germany

\section{Introduction}

Rodent brains compute relationships between motor commands to whisker muscles and signals from whisker mechanoreceptors to infer the position, size and texture of objects in their near environment. These computations are carried out by multi-level sensorimotor neuronal loops (reviewed by Ahissar and Oram 2015) that include the "first-order" ventral posteromedial (VPM) and the "higher order" posterior (Po) thalamic nuclei as well as the whisker domains of the motor (M1wk) and primary somatosensory (S1BF) cortices (Theyel et al. 2010; Sherman and Guillery 2013).

M1wk and S1BF cortices direct exploratory whisker movements (Matyas et al. 2010; Ahissar and Oram 2015). In both areas, pyramidal neurons in layer (L)5 innervating the brainstem face motor and sensory centers send axonal collaterals to Po, where they are able to drive thalamic neurons 
(Urbain and Deschênes 2007; Theyel et al. 2010; Groh et al. 2014; Economo et al. 2018). Po neurons also receive ascending sensory information from the trigeminal nuclei (Veinante and Deschênes 1999; De Chazeron et al. 2004; Guy et al. 2005) and may thus carry out dynamic computations between the motor commands and the ascending sensory signals (Groh et al. 2014). In turn, Po neurons have been shown to monosynaptically excite S1BF pyramidal neurons "in vitro" (Petreanu et al. 2009; Audette et al. 2018) and evoke spike firing "in vivo" (Gambino et al. 2014; Jouhanneau et al. 2014; Mease et al. 2016; Castejon et al. 2016). Although less well characterized, Po inputs to M1wk have been shown likewise to excite cortical neurons "in vitro" (Hooks et al. 2013) and "in vivo" (Casas-Torremocha et al. 2017).

There is growing evidence suggesting that the functional impact of Po inputs reaching M1wk and S1BF might be different. Anatomical studies in rats and mice have shown that Po axons target both deep and superficial layers in S1BF while arborizing mainly in superficial layers in M1wk (Herkenham 1986; Wimmer et al. 2010; Ohno et al. 2012; Hooks et al. 2015). Moreover, functional "in vitro" studies indicate that Po synapses in S1BF activate mainly the basal dendrites of L5a pyramidal cells and, more weakly, the apical tufts of the same cells in L1 (Petreanu et al. 2009; Audette et al. 2018), while preferentially driving L2-3 and L5a neurons in M1wk (Hooks et al. 2013). In rats, we observed marked differences in the impact of Po inputs on tactile information processing in S1BF and M1wk "in vivo". While Po stimulation interfered with the responses in both areas, the effect was layer specific in each area. Interestingly, Po silencing drastically reduced the whisker responses in M1wk cortex but not in S1BF (Castejon et al. 2016; Casas-Torremocha et al. 2017).

It is conceivable that the above differences reflect, at least in part, variations between areas in the number of Po synapses across cortical laminae, in synapse structure and/or in specific postsynaptic transduction mechanisms. In this context, it is of note that Viaene et al. (2011a) reported that the mean size of Po axonal varicosities labeled by tracer injections in the thalamus and measured using light microscopy seems to be smaller in primary somatosensory area (S1) than those in the secondary somatosensory area (S2). Moreover, this study demonstrated that S1 neuron responses to "in vitro" Po stimulation showed paired-pulse facilitation, small initial excitatory postsynaptic potentials (EPSPs), a graded activation profile, and a metabotropic receptor component, suggesting a modulatory role in information processing. In contrast, neurons recorded in S2 L4 showed paired-pulse depression, large initial EPSPs, an all-or-none activation profile, and no metabotropic receptor component, a profile typical of field-defining or "driver" inputs (Viaene et al. 2011a; reviewed by; Sherman and Guillery 2013).
To determine whether structural and/or functional differences exist between Po synapses in M1wk and S1BF, we first measured axonal length and varicosity distribution across cortical layers using stereological methods, and compared the size of Po synaptic boutons using both light and electron microscopies (EM). In addition, we examined the "in vivo" responses to Po electrical stimulation and pharmacologically investigated the glutamatergic receptor mechanisms involved. Our findings show that Po axon terminals are remarkably different both in structure and in functional impact in M1wk and S1BF.

\section{Methods}

\section{Animals}

Experiments were performed in adult (60-105 days old, 25-32 g in weight) male C57BL/6 mice. Five animals were used for axon tracing experiments ( 3 for light microscopy and 2 for EM) and 11 further animals for electrophysiological recordings. Animals were housed under standard colony conditions with food and water ad libitum under a 12-h light/ dark cycle. All procedures involving live animals were conducted at the Autónoma de Madrid University under protocols approved by our University ethics committee and the competent Regional Government agency (PROEX175/16, PROEX189/16), in accordance with the European Community Council Directive 2010/63/UE. Efforts were made to minimize the number of animals required.

\section{Anesthetic procedures}

All surgical procedures were conducted under isoflurane anesthesia (0.5-1\% in oxygen) following induction with a combination of ketamine $(0.075 \mathrm{mg} / \mathrm{g}$ body weight, i.p. $)$ and xylazine $(0.02 \mathrm{mg} / \mathrm{g}$ body weight, i.p. $)$ for anatomical experiments or just a higher dose of isoflurane (3\%) for electrophysiological recordings. During recordings, the appropriate level of anesthesia was monitored by the presence of delta frequency waves $(1-4 \mathrm{~Hz})$ of high amplitude $(>50 \mu \mathrm{V})$ in the field potentials, as well as by the absence of both spontaneous whisker movements and pinch withdrawal reflex. Buprenorphine hydrochloride $(0.075 \mathrm{mg} / \mathrm{kg}$ body weight, s.c.) was administered for post-surgical analgesia in anatomical experiments. At the time of sacrifice, animals were overdosed with sodium pentobarbital $(0.08 \mathrm{mg} / \mathrm{g}$ body weight, i.p.).

\section{BDA axonal tracing experiments}

To anterogradely label the axons arising from populations of Po neurons, animals were positioned in a stereotaxic 
apparatus (David Kopf Instruments, Tujunga, CA, USA) and placed on a water-heated pad at $37^{\circ} \mathrm{C}$. The midline of the scalp was sectioned and retracted, and a small craniotomy was opened. Borosilicate glass micropipettes $(1 \mathrm{~mm}$ outer diameter with internal glass filament; $10-20 \mu \mathrm{m}$ of inner tip diameter; WPI, Sarasota, FL, USA) were loaded with a $3 \%$ solution of lysine-fixable biotinylated dextran amine (BDA) of 10,000 MW (Invitrogen, Carlsbad, CA, USA) in $0.01 \mathrm{M}$ phosphate buffer (PB; $\mathrm{pH} 7.4)$ and stereotaxically positioned into Po following coordinates according to the Franklin and Paxinos (2008) mouse atlas (AP: $-1.8 \mathrm{~mm}, \mathrm{LM}: 1.2 \mathrm{~mm}$; $\mathrm{DV}:-3 \mathrm{~mm}$ ). A positive current of $2 \mu \mathrm{A}$ ( $7 \mathrm{~s}$ on/off cycles) was applied for $10 \mathrm{~min}$ using a Midgard Current Source (Stoelting Co., Wood Dale, IL, USA). The micropipette was then left in place for additional $10 \mathrm{~min}$ before removal and wound closure. After awakening, animals were allowed to survive for 7 days after BDA injections and were then sacrificed as described below.

\section{"In vivo" electric thalamic stimulation and cortical recording}

The animal was placed in a rodent stereotaxic frame as described above. After the scalp incision, lidocaine (1\%) was applied locally. Three small craniotomies were made. The first over the M1wk which was identified based on the literature as the region where minimal intensity stimulation produces selective whisker movements in mice ( $\mathrm{Li}$ and Waters 1991; Brecht et al. 2004; Hooks et al. 2011; Tennant et al. 2011). This first craniotomy was thus made along the border between area AgM/M2 and area AgL/M1 of the frontal cortex (AP: $0.5-1.5 \mathrm{~mm}$, ML: 1-1.5 mm). The second craniotomy was made over S1BF (AP: from -0.4 to $-1.8 \mathrm{~mm}$, ML: $2.5-3.5 \mathrm{~mm}$ ).

The third craniotomy was vertically located over Po (AP: $-1.8 \mathrm{~mm}$, LM: $1.2 \mathrm{~mm}$ ) according to the Franklin and Paxinos (2008) mouse atlas. A stainless steel bipolar electrode $(120 \mu \mathrm{m}$ diameter, WPI) was then vertically lowered into Po aiming at DV $-3.0 \mathrm{~mm}$. Single square pulses were then delivered though the electrode (0.3-ms duration; Cibertec Stimulator, Madrid, Spain) to produce responses in S1BF and M1wk. Current intensity $(20-80 \mu \mathrm{A})$ was adjusted in each experimental case to the double of the minimum required to elicit responses. When delivered through metal electrodes in a gray matter region of a mammalian brain, currents in the above range have been estimated to activate neuronal bodies within a maximal radius of less than 100-400 $\mu \mathrm{m}$ (Ranck 1975), respectively. Given the small dimensions of mouse Po, selective stimulation was performed by aiming the electrode to a central zone of the nucleus, and histologically verifying the electrode tip position after the experiments (Fig. 2b).
We applied a protocol of 50 single pulses at different frequencies $(1,5$ and $10 \mathrm{~Hz})$ separated by 20 -s intervals without stimulation. Also, three paired-pulse stimulus paradigms were used in which four stimuli with the same current amplitude were delivered into Po with an inter-stimulus interval (ISI) of 50, 100 or $300 \mathrm{~ms}$.

Unit recordings were performed through tungsten microelectrodes (2-4 M $\Omega$; WPI) lowered stereotaxically into M1wk or S1BF. Units were recorded throughout the entire cortical depth. For analysis, data were pooled and compared between

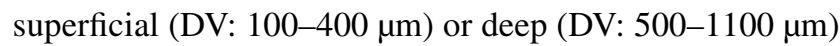
cortical layers in each area. An unanalyzed gap of $100 \mu \mathrm{m}$ was thus left between these compartments as a safeguard against potential errors in laminar localization due to small misalignments or drift of the electrode. In each experiment, we obtained two or three superficial layer recordings and two or three further recordings in deep cortical layers. Recordings were filtered at $0.3-3 \mathrm{kHz}$ and amplified using a DAM80 preamplifier (WPI). Data were recorded continuously, sampled at $10 \mathrm{kHz}$, via an analog-to-digital converter built in to the Power 1401 data acquisition unit (Cambridge Electronic Design, Cambridge, UK) and digitally stored in a computer until further use. Mice were sacrificed with an overdose of anesthetics (see above) immediately after recordings were completed.

\section{Pharmacological blockade of glutamatergic receptors in the cortex}

To examine the various glutamate receptor components involved in cortical responses to Po thalamocortical activation, the NMDA receptor antagonist D-2-amino-5-phosphonopentanoate (D-AP5; $50 \mu \mathrm{M} ; 0.1 \mu \mathrm{l}$; Biogen Científica, Madrid, Spain), the non-selective group I/group II metabotropic glutamate receptor antagonist (+)-alpha-methyl-4-carboxyphenylglycine (MCPG; $10 \mu \mathrm{M} ; 0.1 \mu \mathrm{l}$; Tocris Bioscience, Bristol, UK), and saline (control) were injected into M1wk and S1BF cortices in different animals. These were injected near the location of the tungsten microelectrode in each area (within a $500 \mu \mathrm{m}$ radius to allow diffusion in the recording site). Drugs were slowly delivered through a cannula connected to a Hamilton syringe ( $1 \mu \mathrm{l}$; Bonaduz, Switzerland) over a 1-min period and recordings were performed 10 min after drug application.

\section{Perfusion and tissue processing for optical microscopy}

Animals were first perfused transcardially with $6 \mathrm{ml}$ of saline for $1 \mathrm{~min}$, followed by $50 \mathrm{ml}$ of $4 \%$ paraformaldehyde (PFA; diluted in $0.1 \mathrm{M} \mathrm{PB}, \mathrm{pH}$ 7.4) for $8 \mathrm{~min}$. Brains were then removed from the skull and postfixed overnight at $4{ }^{\circ} \mathrm{C}$ in the same fixative. Subsequently, brains were cryoprotected by 
soaking in $30 \%$ sucrose $\left(0.1 \mathrm{M} \mathrm{PB}, 4{ }^{\circ} \mathrm{C}, 48 \mathrm{~h}\right)$. Two parallel series of coronal sections ( $60 \mu \mathrm{m}$ thick) were obtained on a freezing microtome (SM 2400; Leica, Germany).

In the axonal labeling experiments, sections were processed using avidin-biotin-peroxidase (1:100; ABC, Vectastain Elite, Vector Laboratories, Burlingame, CA, USA) and diaminobenzidine-glucose oxidase, with nickel enhancement (Shu et al. 1988). Serial sections were alternatively counterstained with thionin or cytochrome oxidase histochemistry (CyO; Wong-Riley et al. 1979) to allow a precise cytoarchitectonic localization of the labeling. In the recording experiments, sections were stained only with CyO. Sections were finally mounted onto gelatin-coated glass slides, air dried, dehydrated through an ascending series of ethanol, defatted in xylene for $60 \mathrm{~min}$ and coverslipped with DePex (Serva, Heidelberg, Germany).

\section{Perfusion and tissue processing for EM}

Seven days after the BDA injection, animals were perfused first with saline, and then with $4 \% \mathrm{PFA}+0.1 \%$ glutaraldehyde in $0.1 \mathrm{M}$ PB for $30 \mathrm{~min}$. Brains were then blocked and immersed for $1 \mathrm{~h}$ in $4 \%$ PFA in PB at $4{ }^{\circ} \mathrm{C}$. Serial $50-\mu \mathrm{m}$-thick coronal vibratome sections (Leica VT 1200S) were collected in PB as two parallel series, and subsequently immersed in sucrose ( $30 \%$ in $0.1 \mathrm{M} \mathrm{PB}$ ) overnight.

Both series of sections underwent three consecutive rapid freezing-thawing cycles in liquid nitrogen to increase membrane permeability, and were then processed, in parallel, to develop BDA as described above, with the exception that in the second series, the oxygen-peroxide blocking step and the nickel sulfate enhancement were omitted. The first series was used to localize labeled Po axon arborizations and BDA deposits. The parallel sections from the second series containing the region with the heaviest cortical labeling underwent further processing for EM. Free-floating sections were first incubated for $45 \mathrm{~min}$ in $1 \%$ osmium tetroxide in $0.1 \mathrm{M}$ PB, washed in PB, rinsed in 50\% ethanol, incubated for $40 \mathrm{~min}$ in $1 \%$ uranyl acetate diluted in $70 \%$ ethanol in the dark, and finally dehydrated in an ascending ethanol series to absolute ethanol. The dehydrated sections were transferred to acetonitrile, and then immersed in epoxy resin (Durcupan ${ }^{\mathrm{TM}}$; Electron Microscopy Science, Hartfield, PA, USA) overnight. Finally, sections were flat embedded in Durcupan ${ }^{\mathrm{TM}}$ and polymerized at $60^{\circ} \mathrm{C}$ for $48 \mathrm{~h}$. Under a dissection stereomicroscope, samples containing the appropriate layers in M1wk or S1BF cortices were cut out, and then glued onto pre-polymerized resin blocks.

Embedded tissue blocks were cut on an ultramicrotome (Leica Ultracut UCT) into ultrathin sections $(\sim 60 \mathrm{~nm}$ thickness) and collected on pioloform-coated slot copper grids. Thereafter, they were counterstained with lead citrate (3 min; Reynolds 1963).

\section{Stereological axon measurements}

Stereological analyses were carried out with a BX61 light microscope (Olympus, Japan) equipped with a MT12 microcator $(0.5-\mu \mathrm{m}$ resolution; Heidenhain, Traunreut, Germany), a X-Y-Z high-precision motorized microscope stage (ProScan II; Prior Scientific, Cambridge, UK), and an Olympus DP-71 digital camera connected to a computer running the NewCAST stereology software package (Visiopharm, Hørsholm, Denmark).

In samples from three different Po axon-labeling experiments, absolute axonal length in individual cortical layers of S1BF and M1wk was estimated using the isotropic virtual plane method following a fractionator sampling scheme (Drøjdahl et al. 2010). For that purpose, the contours of the layers within the cortical column where the axonal arborizations were present were drawn for each cortical region with the $4 \mathrm{x}$ objective in the $60-\mu \mathrm{m}$-thick sections immunostained for BDA; then, these areas were sampled by the equidistant and parallel isotropic-randomly oriented-virtual planes which contain three-dimensional 'sampling boxes' inside. The intersections between the planes and the BDA-labeled fibers were counted. We used a distance between virtual planes of $20 \mu \mathrm{m}$, a sampling box volume of $47,020 \mu \mathrm{m}^{3}$ and a step length area of between 10,000 and 22,500 $\mu \mathrm{m}^{2}$ (depending on the axonal density previously observed in each cortical layer). We considered and corrected the inhomogeneous shrinkage in the $z$-axis calculating the numberweighted mean section thickness, as previously described (Bermejo et al. 2003).

In addition, the absolute number of axonal varicosities in each layer was determined with the optical fractionator method (West and Gundersen 1990). Using the 100× oilimmersion objective in the same contours drawn for axonal estimations, swellings larger than twice the thickness of surrounding axon domains were identified as varicosities. Axon enlargements at branching points were not considered. The individual volume of the optical disectors used to sample the regions of interest (cortical layers) was $4704 \mu^{3}$ and the step length area for each layer was the same as for axonal length estimations.

The distribution of axonal length and varicosities per layer was then calculated. The precision of the stereological estimations was calculated by obtaining the coefficient of error due to the sampling method (Gundersen et al. 1999). We aimed a minimum counting of 100 axonal intersections or varicosities per cortical layer to obtain a coefficient of error below 0.1 . In only a few cases, the coefficient of error was higher than 0.1 because the areas were too small or axonal density too low to allow counting enough intersections or varicosities.

In addition, to facilitate comparison with the visual appearance in S1BF of Po arborizations in the radial 
dimension of the cortex, we calculated both "length density" (axonal length per volume) and "density of varicosities" (number of varicosities per volume) in each of the three cases included in the study. For this purpose, we estimated the volume of each layer across the cortical sector containing the entire Po-labeled axon population. This volume was estimated using the Cavalieri principle (Gundersen and Jensen 1987); a point grid with a known area per point was used and the number of points reaching each cortical layer in every section sampled were computed. The volume estimation was then obtained multiplying the number of points, the area per point and the separation between sections. Densities were obtained dividing the previously calculated absolute axonal length or number of varicosities by the volume in each layer.

\section{Light microscopy and EM bouton size analyses}

Under bright-field optics in Nikon Eclipse 600 microscope (Nikon, Tokyo, Japan) the Po axonal arborizations appeared as sharply labeled filaments with frequent varicose swellings. To estimate and compare the size of varicosities (putative synaptic boutons), we measured the maximal projection area from live images using a Nikon DMX1200 camera fitted to the microscope and the NISElements imaging software tools (v3.2; Nikon). To this end, the major perimeter of each varicosity was focused at $1000 \times$ and delineated over the computer screen using the polyline and polygon software tools. For each cortical area and for each animal, 500 randomly selected varicosities were measured. Three animals were analyzed (3000 varicosities in total). We did not include varicosities with cross-sectional areas (maximal projection) near or below the microscope resolution limit $\left(<0.2 \mu \mathrm{m}^{2}\right)$.

The size of Po axonal boutons was estimated and compared also from the area of sectioned profiles on EM micrographs. At the EM level, BDA-labeled Po boutons were easily identifiable by the electron-dense opaque DAB reaction product. For measuring the bouton size, only sections in which a synapse was visible were considered. Sections were examined with a Zeiss Libra 120 transmission electron microscope (Carl Zeiss, Oberkochen, Germany) equipped with a bottom-mounted Proscan 2K digital camera (Fa. Tröndle, Moorenweiss, Germany). Bouton profiles were analyzed on digital images obtained using the Multi-Image Acquisition software (SIS; Olympus, Münster, Germany) at a final magnification of $8000 \times$. For each cortical region, the surface areas of 50 boutons were measured with OpenCAR software as described in detail in Rodriguez-Moreno et al. (2018).

\section{Electrophysiological data analysis}

Single-unit activity was extracted with the aid of Spike2 software (Cambridge Electronic Design) for spike waveform identification and analysis. In some cases, antidromic activity was present, but these records were excluded from the analysis. The sorted spikes were analyzed and quantified using peri-stimulus time histograms (PSTH; bin width $1 \mathrm{~ms}$ ), where time $0 \mathrm{~ms}$ corresponded to the onset for the first stimulus in the stimulation train. The PSTH was calculated from 50 successive Po electrical stimuli. Response magnitude was defined as the total number of spikes per stimulus occurring between stimulus onset and $50 \mathrm{~ms}$ afterwards. To analyze Po-evoked responses during the application of stimulation trains, we calculated the response magnitude variation as the percentage change of the spikes measured in the second (P2), the third (P3) and the fourth (P4) pulses with respect to the first pulse (P1; normalized as $100 \%)$.

Paired-pulse ratios (PPR) were defined as the cortical unit response to $\mathrm{P} 2, \mathrm{P} 3$ or $\mathrm{P} 4$ in any given train divided by the response to P1. Thus, a PPR of 1 reflects no change between pulses; PPR $<1$ indicates depression of the response, and PPR $>1$ indicates facilitation.

Initial (onset) and final (offset) latencies and response duration were measured in the PSTH. Initial latency was considered the time elapsed between stimulus onset and when the number of spikes doubled those in the spontaneous activity period. Final latency was considered the time when the number of spikes decayed to the same level as during the spontaneous activity period.

\section{Statistical analysis}

Statistical analysis was computed using GraphPad Prism 5 software (San Diego, CA, USA) or SPSS Statistics software (v. 23; IBM, Armonk, NY, USA). The threshold level of significance was set at $p<0.05$, indicating this as $(*), p<0.01$ as $(* *)$ and $p<0.001$ as $(* * *)$.

For the stereological dataset, comparison was performed by one-way ANOVA and either Bonferroni or Games-Howell post hoc pair-wise comparison methods, depending on the homogeneity of variances. Varicosity size distribution and medians were compared between pairs of areas/layers with a two-sample Kolmogorov-Smirnov (K-S) test and the Mann-Whitney $U$ test (M-WUT).

For the electrophysiological dataset, first we applied the Shapiro-Wilk normality test. Then, we used the paired Student's two-tailed $t$-test or M-WUT, depending whether the data were normally distributed or not. For multiple comparisons, we used a two-way ANOVA, plus Bonferroni's multiple comparison tests as a post hoc comparison method. 


\section{Results}

\section{The laminar distribution of Po varicosities in S1BF and M1 wk is markedly different}

To examine possible structural differences between Po axonal terminals in S1BF and M1wk, we selectively labeled Po populations of axons by means of iontophoretic BDA injections in the thalamus. Three experiments in which a tracer deposit was confined to Po were analyzed (Fig. 1a). The glucose oxidase BDA-nickel labeling protocol produced a sharp, continuous, Golgi-like axonal labeling. In all injections, axonal arborizations with varicose swellings were clearly identifiable at the light-microscope level (Fig. 1a-f).

In M1wk and S1BF, a marked layer-specific distribution of axonal arborizations was observed (Fig. 1b, c). In general, axonal varicosities at thalamocortical axons, most of which represent synaptic sites (White et al. 2004; Rodriguez-Moreno et al. 2018), were obscured by the mess of labeled axon filaments. Thus, we separately quantified axonal lengths and varicosity numbers with stereological methods in all cortical layers, and compared them between the two areas (Fig. 1b, c; Supplementary materials Table SM1). The axonal length analysis confirmed that while the mean length was virtually identical in layers $1,5 \mathrm{~b}$ and 6 , it was significantly higher by nearly twofold in L5a of S1BF $(48 \% \pm 7.7)$ when compared with M1wk $(23.3 \% \pm$ $7.2 ; p=0.006)$. Conversely, axonal length was lower by threefold in L2-4 of S1BF $(14.9 \% \pm 5.7)$ than in the equivalent layers of M1wk (47.6\% $\pm 7.7 ; p=0.004)$. Differences in the layer-specific distribution of axonal varicosities were even more striking: $56.2 \% \pm 11.2$ were located in L5a of S1BF but only $18.4 \% \pm 7.1$ were observed in the same layer of M1wk $(p<0.001)$. Conversely, while just $12.5 \% \pm 6.4$ Po varicosities were located in L2-4 of $\mathrm{S} 1 \mathrm{BF}$, the equivalent layers of M1wk contained $55.5 \% \pm$ $8.1(p<0.001)$. The sharp inversion observed in the distribution of Po axonal varicosities in L2-4 and L5a of the two areas seems all the more significant in view that distributions in the remaining cortical layers were similar.

\section{Po axonal boutons in M1wk are significantly larger than those in S1BF}

Using high-power light microscopy, Po axonal varicosities in M1wk looked substantially larger than those in S1BF (Fig. 1d, e). To quantitatively assess these differences, we first compared the size of axonal varicosities (maximal projection areas measured at $1000 \times ; 500$ varicosities per experiment, in three experiments; see Methods). We demonstrate that, on average, Po varicosities in M1wk L3-4 were significantly larger $\left(1.6 \pm 0.8 \mu^{2}\right)$ than in those in S1BF L5a $\left(1.01 \pm 0.5 \mu \mathrm{m}^{2}\right.$; M-WUT, $\left.p<0.001\right)$, and had a significantly different frequency in size distribution (K-S, $p<0.001$; Fig. 1f) with a substantial tail of larger boutons.

To further quantify and distinguish between these two populations, we also measured bouton size on a sample of EM single images of BDA-labeled Po synaptic boutons. Surface area measurements confirmed that M1wk L3-4 boutons were significantly larger than those in S1BF L5a $(0.99 \pm 0.5$ $\mu \mathrm{m}^{2}$ vs. $\left.0.67 \pm 0.3 \mu \mathrm{m}^{2} ; \mathrm{M}-\mathrm{WUT}, p<0.001\right)$. Moreover, Po synaptic boutons had a different frequency of size distribution in the above two areas (K-S, $p=0.009$; Fig. 1i). In addition, this analysis showed that, in general, axonal varicosities correspond to asymmetric synaptic sites (Fig. 1g, h) indicative of their glutamatergic nature.

\section{Po synapses in S1BF and M1 wk show different dynamic profiles "in vivo"}

In several glutamatergic projection systems, including thalamocortical axons, differences in synaptic bouton size have been correlated with specific functional properties (reviewed in Sherman and Guillery 2013). These include amplitude changes to repetitive stimulation and the involvement of different receptor mechanisms. To investigate possible functional differences in M1wk and S1BF unit responses to repetitive Po activation, trains of electrical stimulation to Po were applied and recordings were obtained extracellularly in superficial and deep cortical layers of each area (Fig. 2a, b). Spike counts revealed a low spontaneous activity in both superficial and deep neuronal populations in M1wk $(0.6 \pm 0.1$ spikes/s, $n=33$; and $0.4 \pm 0.1$ spikes/s; $n=23$, respectively $)$ and in $\operatorname{S} 1 \mathrm{BF}(0.5 \pm 0.1$ spikes $/ \mathrm{s}, n=32$; and $0.6 \pm 0.1$ spikes/s; $n=21$, respectively).

First, we examined M1wk and S1BF unit responses to electrical Po "in vivo" stimulation at increasing frequencies (1, 5 and $10 \mathrm{~Hz}$; 20-80 $\mu$ A; Fig. 2a). Single-pulse Po stimulation induced orthodromic spike responses in all layers of M1wk and S1BF (Fig. 2c). $1 \mathrm{~Hz}$ single-pulse Po stimulation elicited $1.3 \pm 0.1$ spikes/stimuli in superficial layers and $1.5 \pm 0.1$ in deep layers of M1wk and $1.4 \pm 0.1$ in both S1BF superficial and deep layers. However, no significant differences in spike probability were observed between M1wk and S1BF in either superficial $(p=0.193)$ or deep layers $(p=0.327)$. Units in M1wk superficial layers increased their firing at higher stimulation frequencies $(F=6.68, p=0.002)$, while those in deep layers did not change $(F=1.26$; $p=0.292$; Fig. 2d). In S1BF, neurons recorded in both the superficial and the deep layers increased their firing at higher Po stimulation frequencies $(F=6.42, p=0.002$ and $F=5.40$, $p=0.007$, respectively; Fig. 2d). 

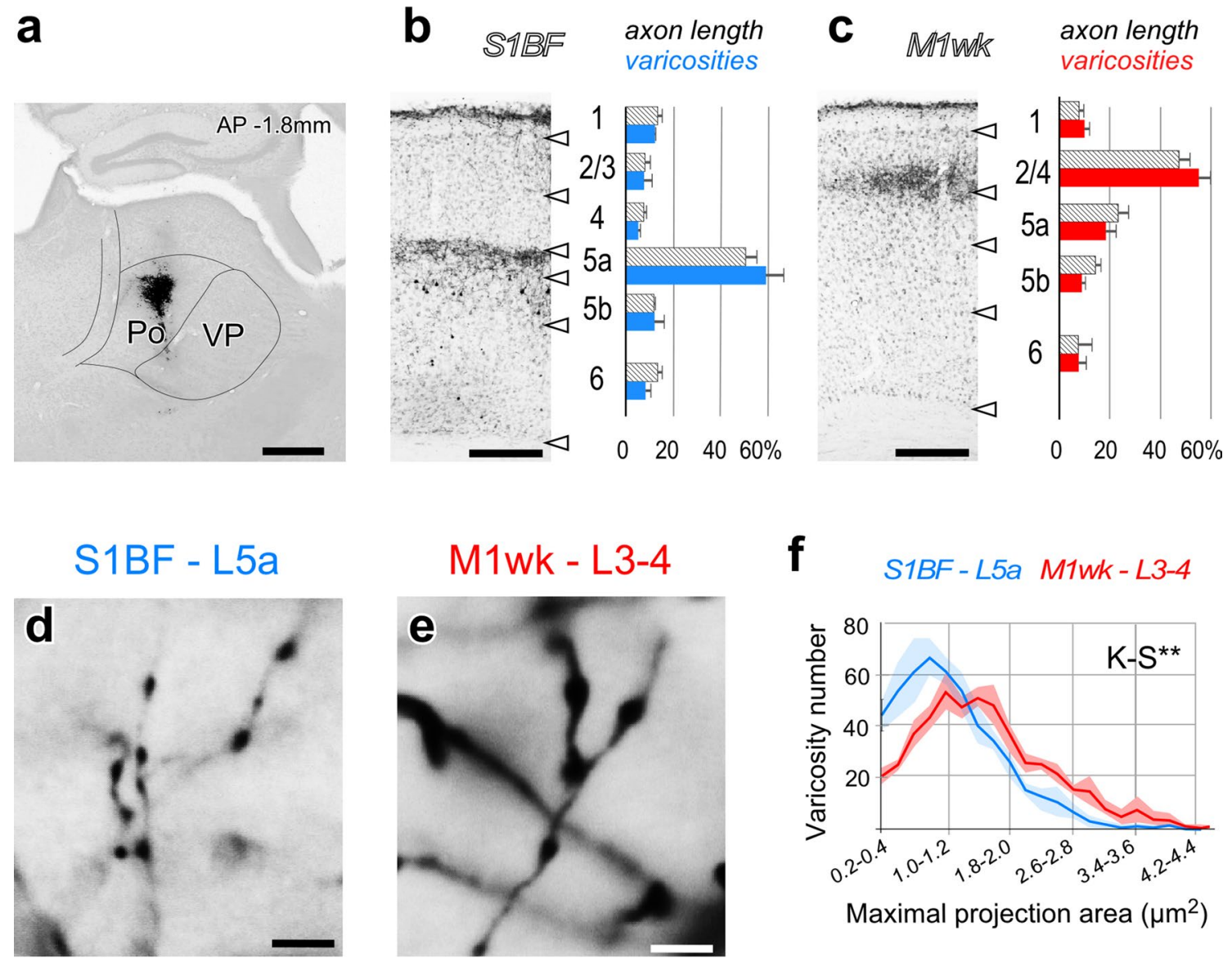

f

S1BF - L5a M1wk - L3-4
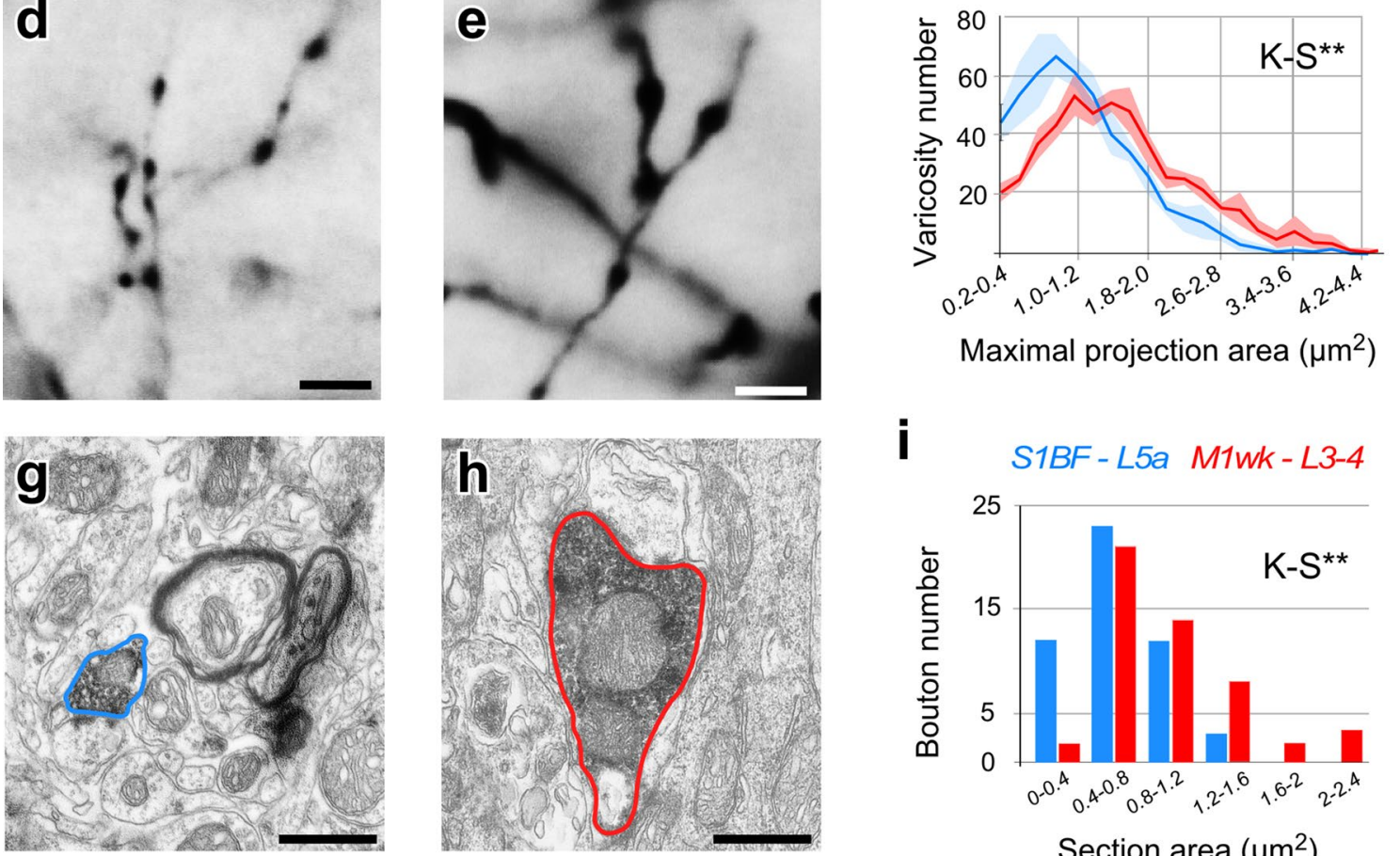

Maximal projection area $\left(\mu \mathrm{m}^{2}\right)$
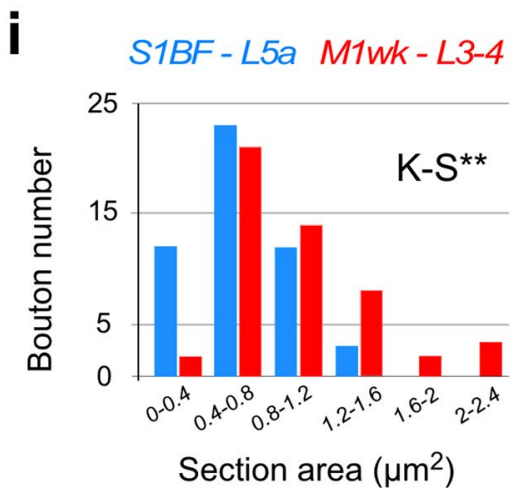

Fig. 1 Quantitative analysis of axonal length, varicosity number and bouton size of Po axons in different layers of areas S1BF and M1wk. a Lightly thionin-counterstained coronal thalamus section showing the center of a representative iontophoretic BDA deposit in Po. The boundaries between Po and ventral posterior complex (VP) are indicated by contour lines. b, $\mathbf{c}$ Stereological estimates of axonal length (hatched bars) and number of varicosities (color bars) per individual cortical layer, as percentage of the total. For reference, a photomicrograph of the axonal BDA labeling in a representative coronal column sample from each area is provided (lightly thionin counterstain). High-magnification light-microscope images of BDA-labeled vari-

In addition, we applied several protocols, each using a different frequency of repetitive Po stimulation to examine short-term changes in unit responses. Four pulses (P1-P4) cose axonal branches in L5a of the S1BF ("d") and L3-4 of M1wk ("e"). Note the differences in the size of varicosities between the two areas. $\mathbf{f}$ Distribution of axon varicosity intervals (measured as maximal projection area) in M1wk L3-4 (blue line) or S1BF L5a (red line). SEM is represented as paler shadings of the same colors. Lowpower EM images of BDA-labeled Po boutons in S1BF L5a ("g") and M1wk L3-4 ("h"). Labeled bouton profiles are outlined in the same colors as above. i Range of Po bouton cross-sectional area measured on EM sections. Scale bars a $500 \mu \mathrm{m} ; \mathbf{b}, \mathbf{c} 250 \mu \mathrm{m}$; d, e $5 \mu \mathrm{m} ; \mathbf{g}$, h $1 \mu \mathrm{m}$

spaced at different intervals (ISI) of 50, 100 or $300 \mathrm{~ms}$ were applied in each protocol (Fig. 3a).

Besides receiving inputs from Po, M1wk and S1BF are known to be heavily linked by cortico-cortical connections 


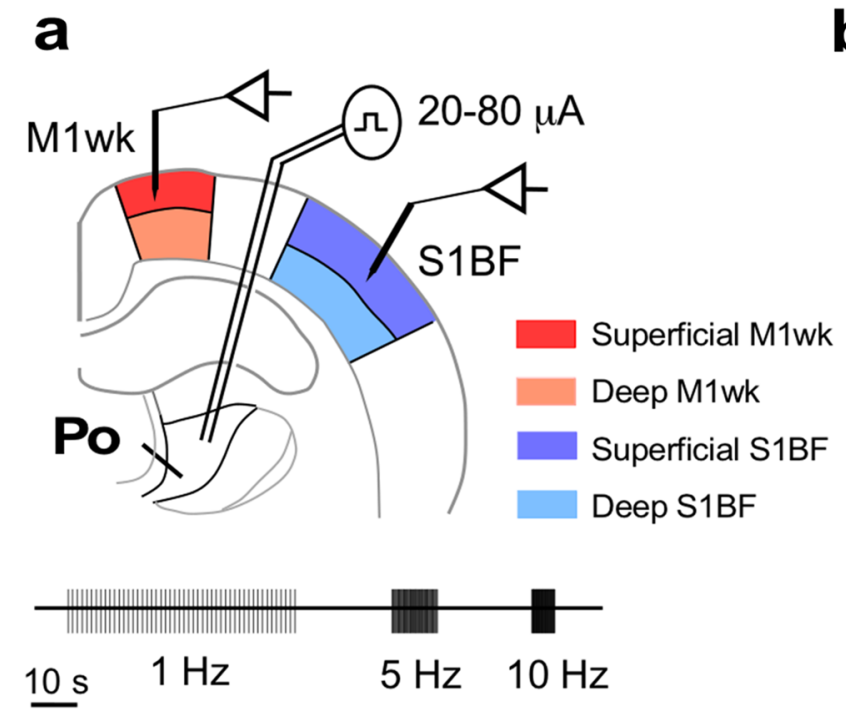

b
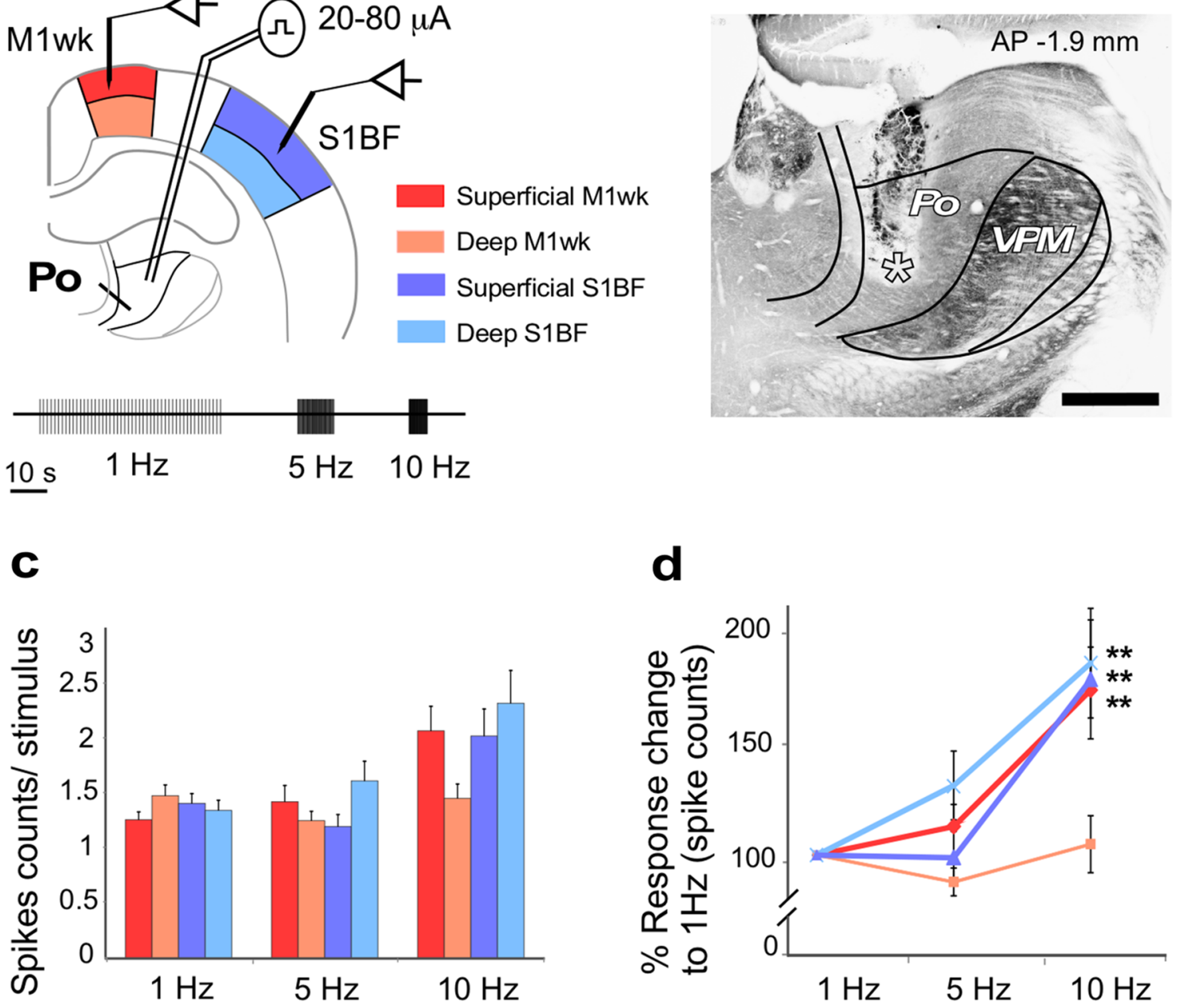

Fig. 2 M1wk and S1BF unit responses to Po "in vivo" stimulation at different frequencies. a Schematic drawing of the experimental design. Electrical pulses $(0.3 \mathrm{~ms}, 20-80 \mu \mathrm{A})$ were applied to Po and unit responses were recorded in S1BF or M1wk cortices. Units recorded in superficial or deep cortical layers were indicated by different colors. A protocol of 50 single pulses delivered at three different frequencies $(1,5$ and $10 \mathrm{~Hz})$ separated by 20 -s pause intervals was applied. b Representative coronal section of the thalamus show-

(Mao et al. 2011; Hooks et al. 2013). As a way to differentiate between the direct effects of Po inputs to both areas vs. the effect of connections between the areas, we examined the onset and offset latencies and the response duration to P1 in ISI 50-ms protocol (Fig. 3b). This analysis showed that the first activation (onset latency) in M1wk and S1BF was almost simultaneous $(p=0.631)$. First responses consistently appeared in S1BF deep layers $4.6 \pm 0.2 \mathrm{~ms}$ after Po stimulation $(p=0.005)$, then $5.6 \pm 0.3 \mathrm{~ms}$ in S1BF superficial layers and $5.8 \pm 0.3 \mathrm{~ms}$ in M1wk superficial layers. Finally, responses occurred with a slightly longer latency in the deep layers of M1wk $(6.9 \pm 0.4 \mathrm{~ms} ; p=0.027)$. No differences

ing the trace of the bipolar electrode in Po. Tip position is indicated by an asterisk. Cytochrome oxidase counterstain. Bregma anteroposterior level (AP) is indicated. VPM: ventral posteromedial nucleus. Bar $500 \mu \mathrm{m}$. S1BF and M1wk unit responses to Po stimulation at different frequencies. In "c", spike counts per stimulus are compared. In "d", the percent of change at 5 and $10 \mathrm{~Hz}$ is compared to values recorded at $1 \mathrm{~Hz}$

were found in response durations between M1wk and S1BF ( $p=0.089$ in superficial layers, $p=0.329$ in deep layers).

The above four-pulse Po stimulation trains elicited a marked facilitation of unit responses in the superficial layers of M1wk (Fig. 3c). In each train, spike count increases were evident already for the second pulse (P2), and became even more pronounced following the third (P3) and fourth (P4) pulses. At 50-ms inter-stimuli intervals $(F=15.17$, $p<0.001)$ spikes at $\mathrm{P} 4$ increased by nearly threefold when compared with spikes counted after P1 $(p<0.001)$. A weaker facilitation effect was observed with 100 -ms inter-stimuli intervals $(F=5.97 ; p=0.010)$. No change was observed 
a

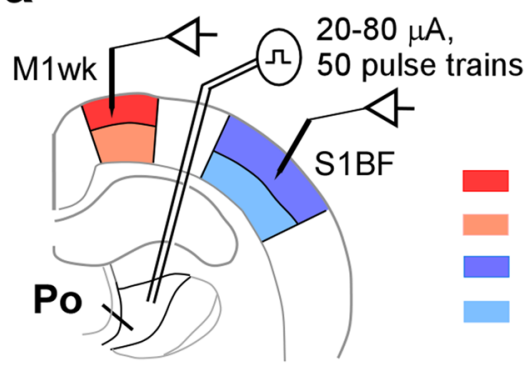

Superficial M1wk

Deep M1wk

Superficial S1BF

Deep S1BF

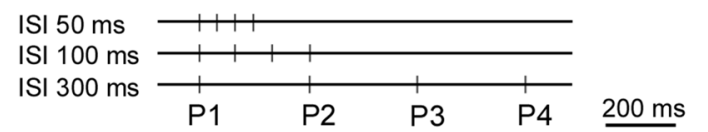

b
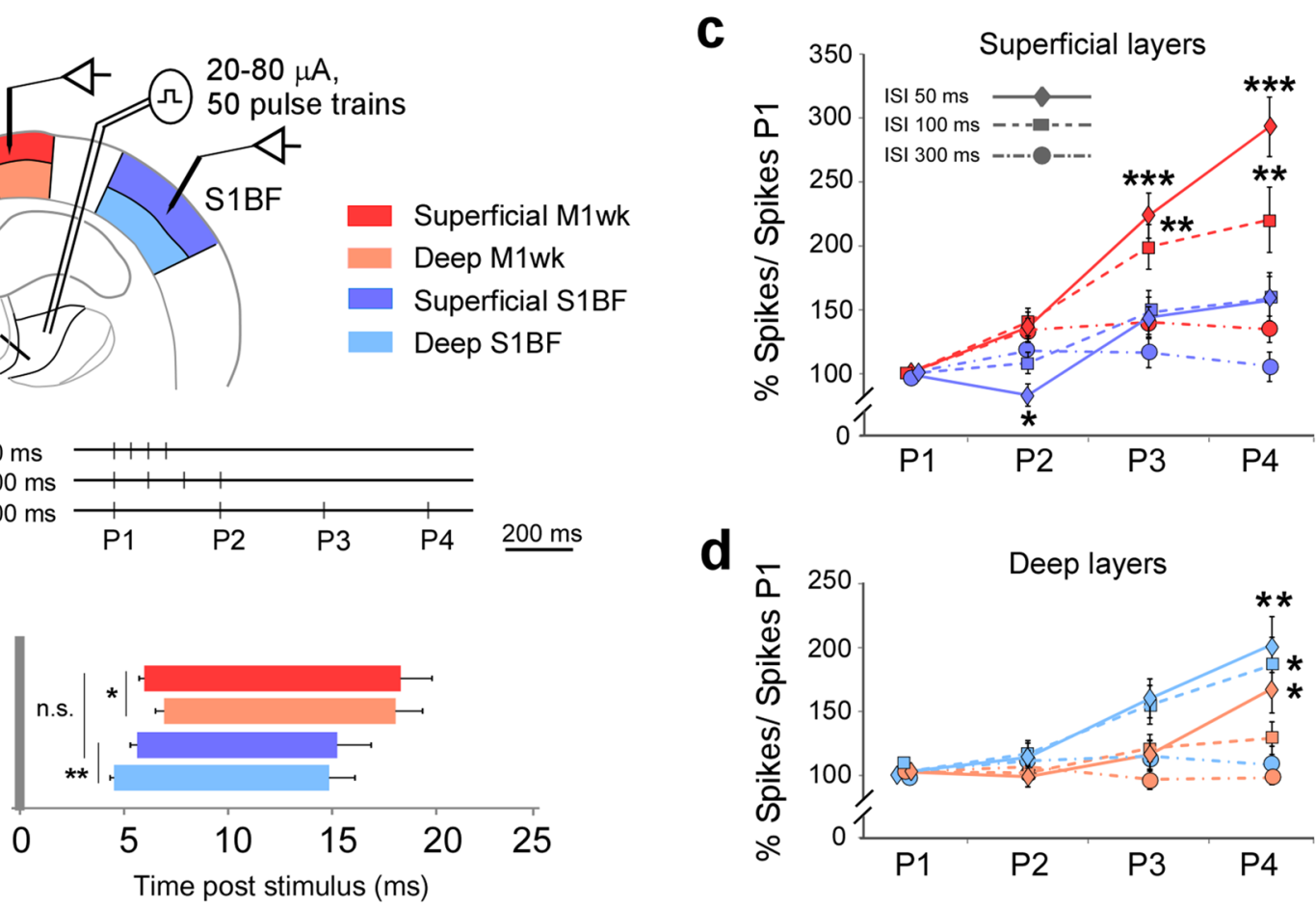

d

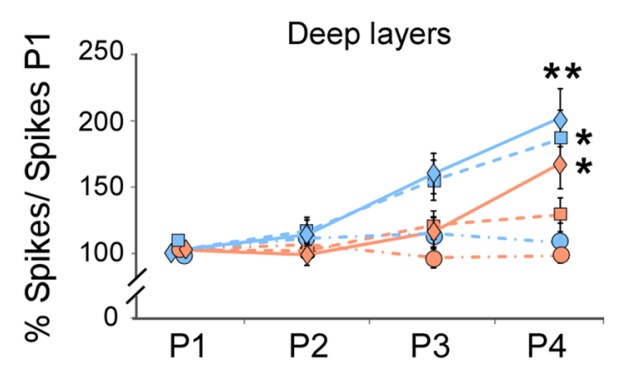

e

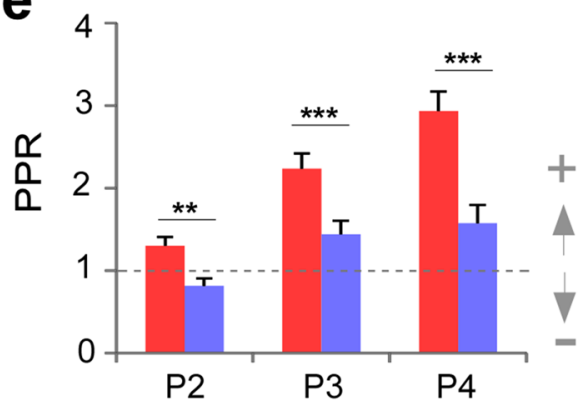

f

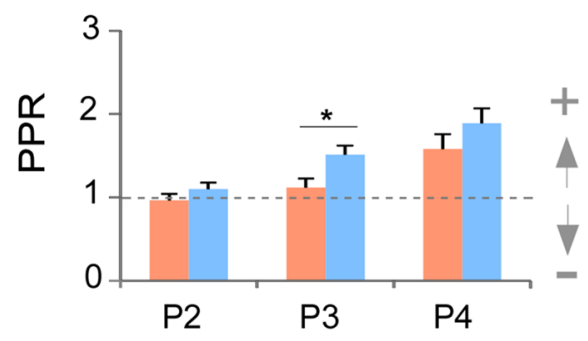

g

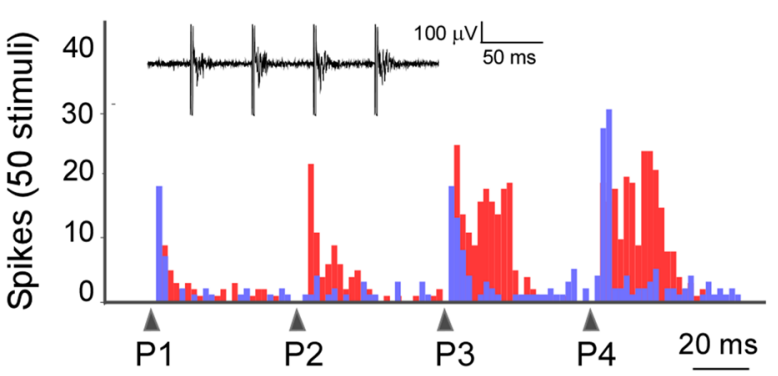

h

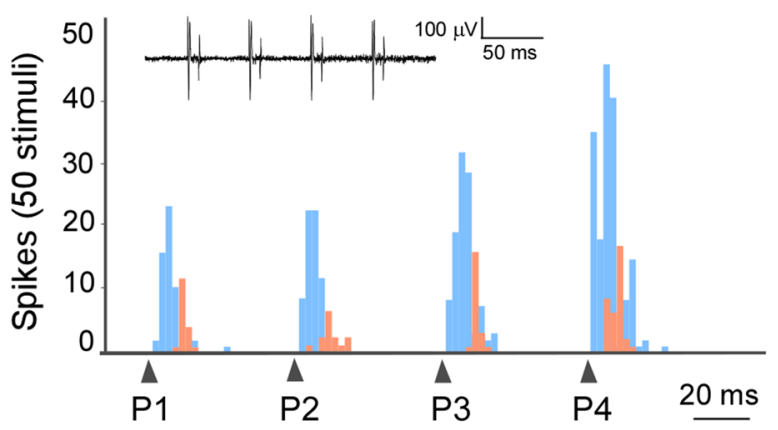

Fig. 3 M1wk and S1BF unit responses display markedly different temporal profiles to repetitive Po stimulation. a Experimental design. Electrical pulses were applied to Po and unit responses were recorded in the cortex. Three different electrical stimulation protocols were applied, consisting each of 50 trains of current pulses delivered every $3 \mathrm{~s}$. Four pulses (P1-P4) were delivered; for each protocol the pulses were spaced at progressively longer intervals (ISI; 50, 100 or $300 \mathrm{~ms}$ ) between them. b Unit response latencies and durations to Po electrical stimulation ( $\mathrm{P} 1,50$ stimuli). Mean onset and offset latencies are represented as horizontal bars. The vertical gray bar indicates stimuli application. c, d Changes in spike counts are represented as the percentage of spikes counted after each pulse (P1-P4) divided by spikes after P1. Counts obtained in each of the above three protocols are represented by lines with different dashes. Counts in superficial ("c") or deep ("d") layers in both areas are shown. Color coding as in panel "a". e, f Paired-pulse ratios (PPR) represented as spike counts after every pulse divided by spike counts in the first pulse (P1). $\mathbf{g}, \mathbf{h}$ An example of M1wk and S1BF unit responses with the 50-ms interpulse protocol. Peri-stimulus time histogram (PSTH) represents spike counts in superficial ("g") and deep ("h") layers. Arrowheads indicate pulse application. Representative spike traces are shown inside PSTHs 
when pulses were separated by $300 \mathrm{~ms}(F=1.54, p=0.207)$. In contrast, using the same Po stimulation protocol (ISI $50 \mathrm{~ms}$ ), unit responses were significantly decreased after $\mathrm{P} 2$ in S1BF superficial layers (a decrease from $1.3 \pm 0.13$ spikes/stimuli in $\mathrm{P} 1$ to $1.0 \pm 0.12$ in $\mathrm{P} 2 ; p=0.047$ ), and no changes were observed after $\mathrm{P} 3$ or $\mathrm{P} 4$, nor during the 100 or $300 \mathrm{~ms}$ ISI protocols (Fig. 3c). Statistical comparison between response changes in superficial layers of M1wk and S1BF during the 50-ms and the 100-ms ISI protocols further indicated that, as a population, neurons in each area responded in significantly different ways (M-WUT, $p<0.001$ and $p=0.049$, respectively).

Although less striking, changes in spike counts during the four successive pulses of Po stimulation were also observed in the deep layers of both areas (Fig. 3d). In M1wk, only a small facilitation of the $\mathrm{P} 4$ response with the 50-ms ISI protocol was observed $(F=2.78, p=0.046)$. In S1BF, response facilitation was observed with both 50 - and 100-ms ISI protocols $(F=6.22, p=0.001$ and $F=3.82, p=0.013$, respectively). Statistical comparison between response changes in deep layers during the 100-ms ISI protocol demonstrated that neurons in each area, as a population, displayed significantly different temporal response profiles $(p=0.026)$.

Analysis of average paired-pulse ratios for the ISI 50-ms protocol confirmed the statistical significance of the above differences (Fig. 3e, f). Examples of representative M1wk and S1BF cell recordings are shown in Fig. $3 \mathrm{~g}$ (superficial layers) and $3 \mathrm{~h}$ (deep layers). In superficial layers (Fig. 3e), this ratio was higher in $\mathrm{M} 1 \mathrm{wk}$ than in S1BF in all single pulses ( $\mathrm{P} 2, p=0.001 ; \mathrm{P} 3$ and $\mathrm{P} 4, p<0.001)$. Remarkably, in $\mathrm{S} 1 \mathrm{BF}$ superficial layers the ratio was $0.8 \pm 0.09$ in $\mathrm{P} 2$, indicative for depression of the response. In deep layers of both areas (Fig. 3f), this ratio is higher in S1BF (P3, $p=0.013$ ) and all ratios indicated facilitation.

\section{Different glutamate receptor types mediate responses in Po synaptic boutons in S1BF and M1wk}

To investigate whether the different response profiles of S1BF and M1wk cells reflect the involvement of specific glutamate receptor types at Po synaptic boutons in each area, the effect of different glutamate receptor antagonists on unit response profiles was performed. Using the ISI 50-ms train stimulation protocol, responses in each area after a local injection of saline (control), D-AP5 (a competitive NMDA antagonist) or MCPG (a non-selective group I/group II metabotropic glutamate receptor antagonist; Figs. 4a, 5a) were compared.

In S1BF (Fig. 4), the facilitatory effects to Po stimulation observed in controls were significantly reduced by D-AP5 in both superficial (P3, $p=0.013 ; n=24$; green columns in Fig. 4b, c) and deep (P4, $p=0.034 ; n=14$; Fig. 4d) cortical layers. Moreover, D-AP5 reversed the spike count decrease observed in $\mathrm{P} 2$ in S1BF superficial layers $(p<0.001$; Fig. 4c). D-AP5 also reduced response duration in both superficial (P2, $p=0.007 ; \mathrm{P} 3$ and $\mathrm{P} 4, p<0.001 ;$ Fig. $4 \mathrm{e})$ and deep layers $(\mathrm{P} 1, p=0.011 ; \mathrm{P} 2, p=0.034 ; \mathrm{P} 3-\mathrm{P} 4, p<0.001$; Fig. 4f). Likewise, responses in S1BF were strongly affected by MCPG. In superficial layers the facilitation observed in control condition was reduced ( $\mathrm{P} 3, p=0.039 ; n=28$; yellow columns in Fig. 4b, c). In deep layers, MCPG completely reversed facilitation, producing a marked decrease of the responses after all pulses (P2-P4, $p<0.001 ; n=15$; Fig. 4d). Response duration was also shortened by MCPG (superficial layers P1-P4, $p<0.001$; Fig. $4 \mathrm{e}$; and deep layers P1, $p=0.005$ and $\mathrm{P} 2-\mathrm{P} 4, p<0.001$; Fig. 4f). Taken together, these observations strongly suggest that both ionotropic NMDA and metabotropic glutamate receptors are involved in response behaviors to Po inputs in S1BF.

In M1wk (Fig. 5), the robust facilitation of cell firing in superficial layers elicited by repetitive Po stimulation observed in controls was partially blocked by D-AP5 (P3, $p=0.037$; $4, p=0.004 ; n=32$; green columns in Fig. 5b, c). Responses after every pulse were shortened (P1, $p=0.006 ; \mathrm{P} 3, p=0.001 ; \mathrm{P} 2$ and $\mathrm{P} 4, p<0.001)$. However, no spike count changes were observed following the application of MCPG in this area (P2, $p=0.452 ; \mathrm{P} 3, p=0.714 ; \mathrm{P} 4$, $p=0.528 ; n=28$; yellow columns in Fig. 5b, c). Moreover, no consistent changes were observed in response duration (only a small decrease after P2, $p=0.005$; Fig. 5d). Overall, these observations seem to indicate that ionotropic NMDA receptors mediate the responses to Po activation in M1wk and that metabotropic glutamate receptors play virtually no role in them.

\section{Discussion}

Our results reveal striking differences between the vibrissarelated domains of the motor and primary somatosensory cortices regarding the layer distribution and size of thalamocortical Po synaptic boutons, the dynamic response profiles of cortical neurons to Po activation, and the glutamate receptor types that mediate these responses.

\section{Po synaptic inputs to the vibrissal motor or sensory cortices preferentially target different layers}

The percent distribution of Po axonal length in S1BF we report here is congruent with our previous observations in a study that examined changes in response to infraorbital nerve lesions (Frangeul et al. 2014). It is also overall consistent with previous non-quantitative observations in rat using bulk (Herkenham 1986; Koralek et al. 1988; Chmielowska et al. 1989; Lu and Lin 1993; Wimmer et al. 2010) or singleaxon anterograde labeling methods (Deschênes et al. 1998; 
a
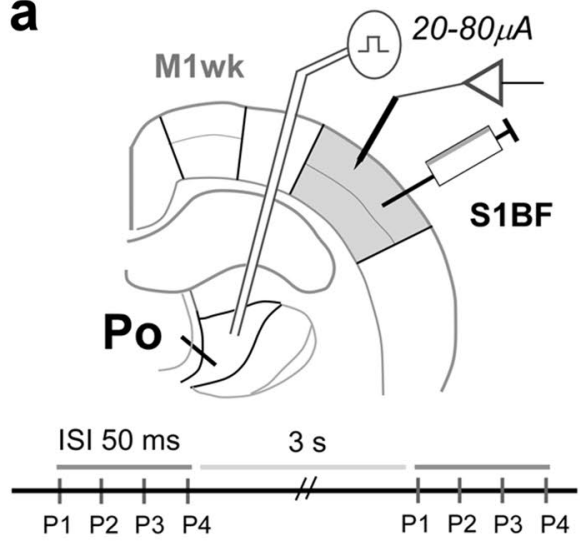

Superficial S1BF

C

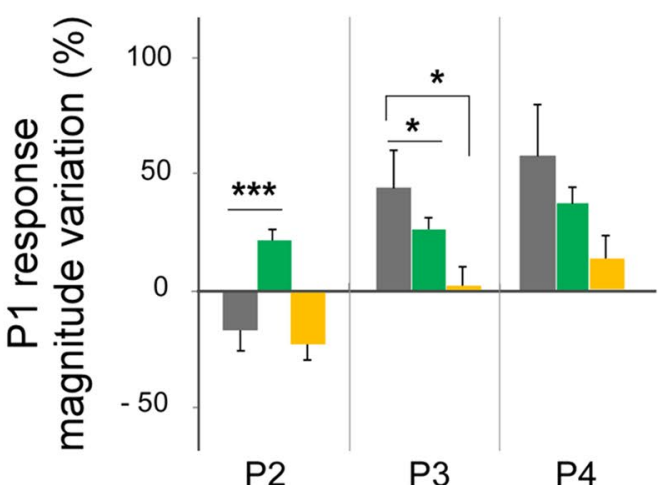

e

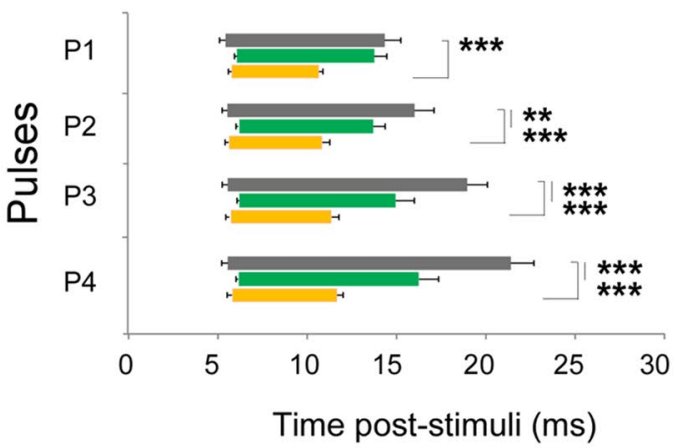

Fig. 4 Pharmacological analysis of glutamatergic receptor systems involved in the "in vivo" S1BF responses to Po activation. a Experimental design. Ten minutes after an injection in S1BF of either the NMDA receptor antagonist D-AP5, the metabotropic receptor antagonist MCPG or a saline control, a four-pulse stimulation protocol (ISI $50 \mathrm{~ms}$ ) was delivered to Po, and unit responses were recorded in the injected area. b Three representative single-unit response profiles (superficial layers of S1BF). Raster plots (top row) or PSTH (bot-

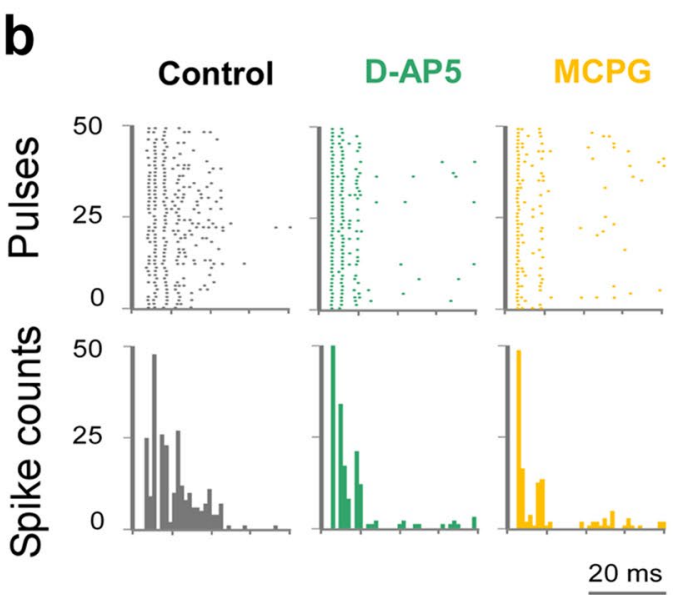

\section{Deep S1BF}

d

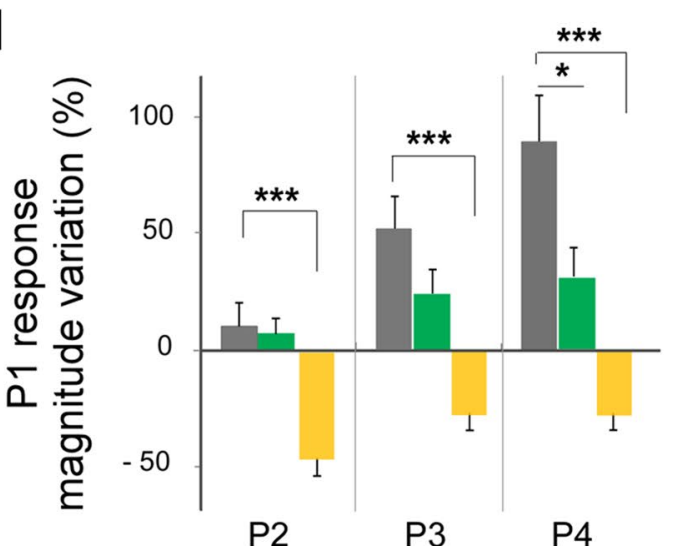

f

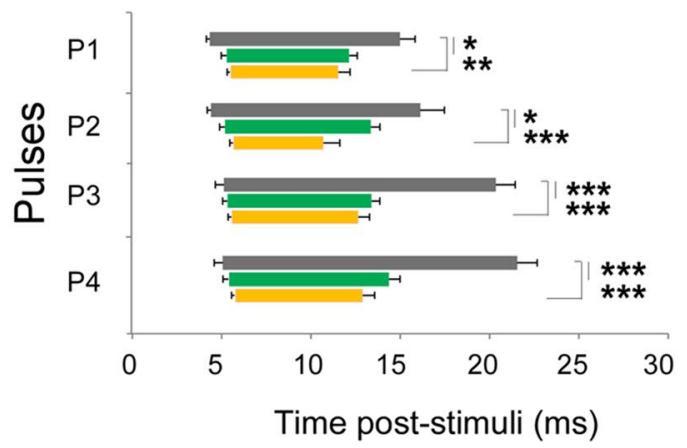

tom) obtained under each drug condition. Gray: saline; green: D-AP5; yellow: MCPG; the same color coding is used in the panels below. Unit response variation in S1BF superficial ("c") or deep ("d") layers represented as the difference (\%) between spikes after P2, P3 and P4 compared with those counted after P1. e, f Latency and duration of unit responses in superficial ("e") and deep ("f") S1BF layers. Poststimulus intervals between mean onset and offset latencies are represented as horizontal bars 

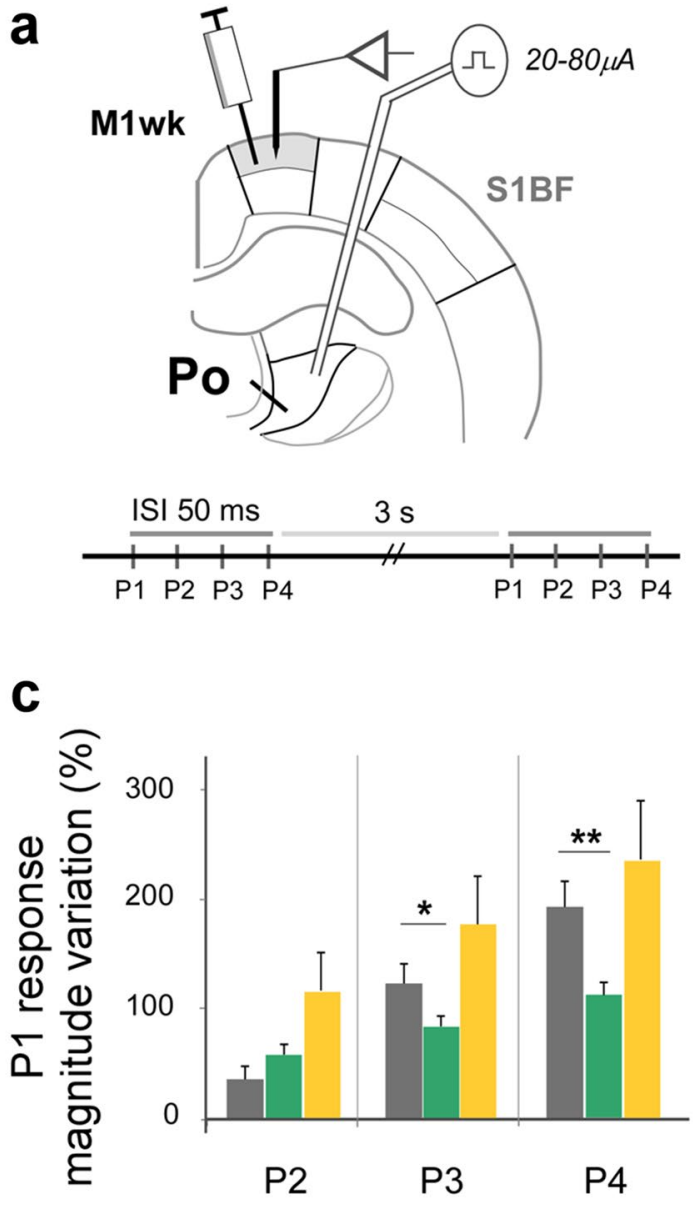

Fig. 5 Pharmacological analysis of glutamatergic receptor systems involved in "in vivo" M1wk responses to Po activation. a Experimental design. Ten minutes after an injection in M1wk D-AP5, MCPG or saline (control), a four-pulse stimulation protocol (ISI $50 \mathrm{~ms}$ ) was delivered to Po, and unit responses were recorded in the injected area. b Three representative single-unit response profiles in M1wk superficial layers. Raster plots (top row) or PSTH (bottom) obtained under each drug condition. Gray: saline; green: D-AP5; yellow: MCPG; the

Noseda et al. 2011; Ohno et al. 2012) which noted that Po axons in S1BF arborize preferentially in layers 5 and 1 . Our data indicate that a large majority of Po arborizations in S1BF are located in L5a. The appearance of heavy axon labeling in L1 of S1BF (Fig. 1b, see also Meyer et al. 2010) thus seems to reflect, to a large extent, the tight packing of a relatively few axonal branches within the small volume of subpial layer 1 . To confirm this interpretation, we calculated axonal length density (length/volume) in S1BF for each of the three BDA labeling experiments analyzed in this study. This revealed evident density peaks in both L5a and L1 in all cases (Supplementary Materials Fig. SM1). Besides, it is to be noted that our BDA deposits were limited to the rostral half of Po, because this is the region that preferentially innervates M1wk. The single-neuron axon

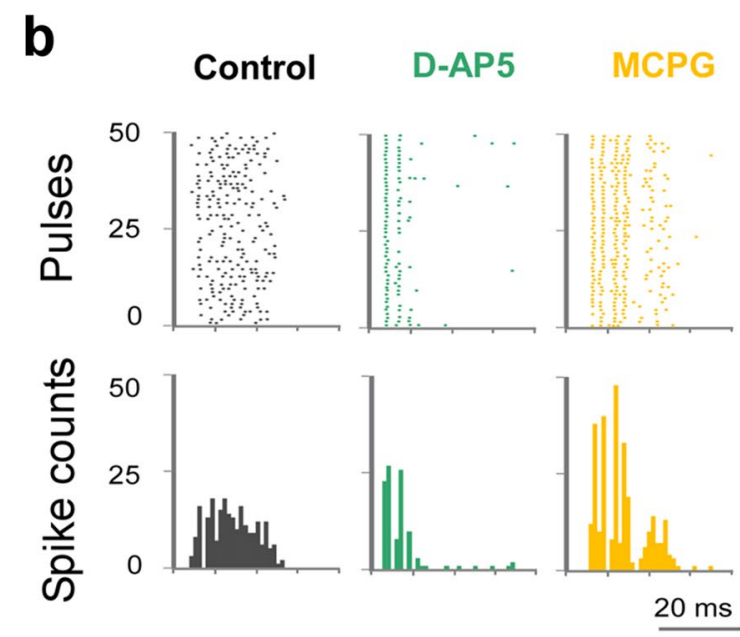

d

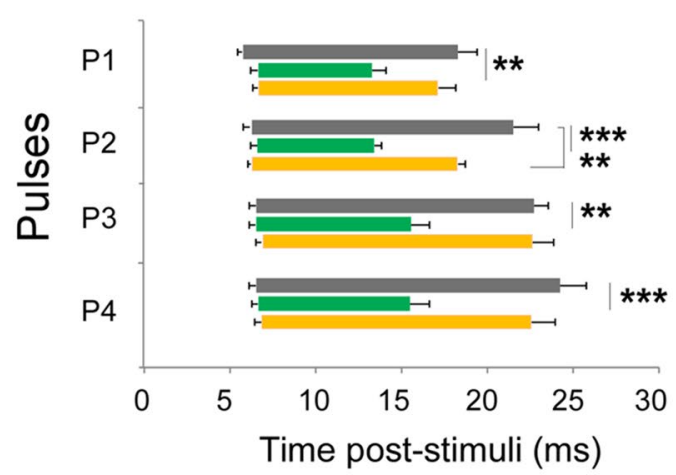

same color coding is used in the panels below. c Unit response variation in M1wk superficial layers. Variation is represented as the difference (\%) between spikes after P2, P3 and P4 compared with those counted after P1. d Latency and duration of unit responses in superficial M1wk layers elicited by Po activation. Post-stimulus intervals between mean onset and offset latencies are represented as horizontal bars

arborization measurements in rats of Ohno et al. (2012) have shown that rostral Po neurons arborize mostly in L5a, while caudal Po/triangular nucleus neurons arborize preferentially in L1. Studies of Po axon distribution in rodent motor cortex (Herkenham 1986; Deschênes et al. 1998; Ohno et al. 2012; Yamawaki et al. 2014) are consistent with our finding that layers 4-3 are the main target of Po axons (Fig. 1; Supplementary Materials Table SM1).

The number and layer distribution of axonal varicosities in thalamocortical axons are of functional relevance, as varicosities have been shown to correlate closely with synaptic sites in these axons (White et al. 2004; Rodriguez-Moreno et al. 2018). Here, we demonstrate that more than half of Po synaptic boutons in S1BF are located in L5a, and less than $13 \%$ in L1. As with axon branch lengths, the analysis of the 
density of varicosities in S1BF revealed also a peak in L1, since the Po axonal arborizations in this layer are spatially concentrated in a narrow subpial band (Fig. SM1). These results are in agreement with a densitometric fluorescence estimation of Po synapse numbers across a S1BF column of GFP-tagged synaptophysin (Meyer et al. 2010), as well as with the finding that S1BF L5 neurons are much more powerfully excited by the selective optogenetic activation of Po axon terminals in L5a than by those in L1 (Petreanu et al. 2009). No study had previously examined Po axon varicosity distribution in M1wk.

Given the highly layer-specific arrangement of neurons and dendrites in the neocortex, the observation that Po axons exhibit marked differences in their layer distribution strongly suggests possible differences in the postsynaptic targets of these axons between S1BF and M1wk. It must be noted that the cortico-cortical "feed-forward"/"bottomup" S1BF axons innervating M1wk arborize in roughly the same layers than Po synaptic boutons do (Mao et al. 2011; Petrof et al. 2015; Hooks et al. 2015). In turn, the M1wk to S1BF cortico-cortical axons, which are thought to provide "feedback"/"top-down" information, arborize in largely the same layers than Po synaptic boutons in S1BF (Johnson and Burkhalter 1996; Crick and Koch 1998; Mao et al. 2011). The contrasting laminar distribution of Po axons in M1wk and S1BF might, therefore, reflect a more general wiring pattern of cortical circuits, and be related to the different hierarchical levels of each area within the cortico-thalamocortical information-processing loops that control whisking (Ahissar and Oram 2015).

\section{Po synaptic boutons are larger in the vibrissal motor cortex than in the somatosensory barrel cortex}

Using both light and electron microscopies, we demonstrate that Po synaptic boutons in S1BF L5a are, on average, significantly smaller than those in M1wk L3-4. Previous light microscopic estimations of Po varicosity sizes either in S1BF (labeled using BDA-ABC after a BDA injection in Po; Viaene et al. 2011a) or in M1 (labeled using GFP florescence after a viral vector injection in Po; Mo and Sherman 2019) are consistent with our observations. It is also interesting to note that present light microscopic measurements of maximal projection area of Po varicosities in L3-4 M1wk $\left(1.6 \pm 0.8 \mu \mathrm{m}^{2}\right)$ are close to that of our VPM thalamocortical varicosities in the L4 S1BF barrels $\left(1.8 \pm 0.8 \mu \mathrm{m}^{2}\right.$; Rodriguez-Moreno et al. 2018). Moreover, the finding that M1wk L4 Po boutons are comparatively large is unexpected, because motor cortex L4 thalamocortical boutons (which arise from both Po and from the ventrolateral nucleus) reportedly are, as a whole, smaller than L4 of S1BF VPM boutons (Bopp et al. 2017).
Studies in different glutamatergic forebrain projection systems, including thalamocortical axons, have shown that bouton size correlates with specific structural and functional synaptic properties. Larger synaptic boutons contain more mitochondria contributing to a larger mitochondrial volume, larger presynaptic vesicle pools, and more extensive and complex active zones (Rollenhagen et al. 2015; Bopp et al. 2017, Hsu et al. 2017; Rodriguez-Moreno et al. 2018; Yakoubi et al. 2018). These features all contribute to increase synaptic neurotransmitter release probability and strength (Matz et al. 2010; Holderith et al. 2012; Bourne et al. 2013; Rollenhagen et al. 2018). As a result, signal transduction in larger synaptic boutons may be both more effective and temporally more precise than that in smaller boutons (Sherman and Guillery 2002, 2013).

\section{Po synapses in S1BF and M1 wk show marked differences in functional dynamics}

Low-intensity electrical stimulation of the rodent thalamus as used here has been shown to activate cortical neurons through thalamocortical synapses (Rose and Metherate 2001; Castejon et al. 2016). Po nucleus stimulation elicited similar orthodromic responses in superficial and deep layers of both M1wk and S1BF. These findings are consistent with "in vitro" (Bureau et al. 2006; Petreanu et al. 2009; Viaene et al. 2011a) and "in vivo" (Gambino et al. 2014; Jouhanneau et al. 2014; Castejon et al. 2016; Mease et al. 2016) observations showing that rodent Po synapses elicit EPSPs in and/or spikes in S1BF pyramidal cells. Moreover, we show that mouse Po neurons are also able to drive cells in superficial and deep M1wk layers, registered with recent rat "in vivo" and mouse "in vitro" studies (Casas-Torremocha et al. 2017; Hooks et al. 2013; Mo and Sherman 2019).

Remarkably, we found that first-unit responses elicited by Po stimulation in S1BF and M1wk are virtually simultaneous. Unit activity in S1BF appears only $1.3 \mathrm{~ms}$ earlier than in M1wk. This minimal delay is compatible with a direct monosynaptic effect in both areas, considering that thalamocortical axons are fast conducting $(\sim 1 \mathrm{~m} / \mathrm{s})$ and that the two areas are at least $3 \mathrm{~mm}$ apart (Franklin and Paxinos 2008). Some previous studies in rat using sensory stimuli suited to drive Po cells (multi-whisker mechanical or whisker pad electrical stimulation; Farkas et al. 1999; Chakrabarti et al. 2008; Casas-Torremocha et al. 2017) noted that responses in M1wk and S1BF showed similar time courses. In contrast, the controlled mechanical stimulation of a single-whisker (which selectively activates VPM axons, but not Po axons) produces first a wave of activity in S1BF and only $8 \mathrm{~ms}$ later a wave of activation in M1wk, which is mediated by corticocortical connections (Ferezou et al. 2007).

The near-simultaneity of the first responses in M1wk and S1BF is entirely consistent with single-cell anatomical 
observations showing that Po signals may reach different cortical areas thought branched axons (Deschênes et al. 1998; Ohno et al. 2012) although it is not yet clear to which extent structurally different Po synaptic boutons arise from specific subpopulations of Po cells or from branched axons of the same neurons targeting both areas (Clasca et al. 2016).

In addition, we show that the neuron response profiles are markedly different in superficial vs. deep layers of both areas. Superficial neurons of M1wk and S1BF, as well as deep S1BF cells increase their firing rate to current pulse trains delivered at progressively higher frequencies, while M1wk deep neurons do not change their spiking behavior (Fig. 2). Likewise, repetitive single-pulse stimulation reveals divergent dynamic response profiles (Fig. 3) resulting in a strong facilitation in M1wk superficial layers but not in S1BF. Firing rates in superficial S1BF neurons even show a significant decrease after the second pulse. Such area- and layer-specific shifts in the global excitation/inhibition balance may reflect several factors. The larger glutamatergic boutons in M1wk are likely to have higher release probability (Viaene et al. 2011a; Ermolyuk et al. 2012; Bourne et al. 2013; Bickford 2016; Rodriguez-Moreno et al. 2018). Moreover, there is recent electrophysiological evidence that Po boutons target specific types of GABAergic interneurons in different layers in S1BF (Castejon et al. 2016; Audette et al. 2018; Williams and Holtmaat 2018). Thus, differences in synaptic bouton distribution across cortical layers may imply different interactions in each area. For example, recent EM data suggest that thalamocortical synapses onto GABAergic interneurons in L4 of the motor cortex may be rare or absent (Bopp et al. 2017).

On the other hand, adaptation is an ubiquitous property of forebrain sensory-motor feedback loops (Ahissar et al. 2000; Maravall et al. 2007). Response depression to repetitive stimulation of sensory organs is the most common form of adaptation observed in these loops. In the somatosensory system, most studies observed robust depression in different layers of S1BF to passive single and multi-whisker stimulations (Ahissar et al. 2000, 2001; Chung et al. 2002; Martin-Cortecero and Nuñez 2014); however, response facilitation has been reported in some studies (Brecht and Sakmann 2002; Garabedian et al. 2003). By comparing S1BF unit responses to electrical activation of the facial nerve that moves the whiskers ("active whisking") vs. the same paradigm of "active whisking" and an additional contact of the vibrissa with an object ("touch"), Derdikman et al. (2006) concluded that adaptation in S1BF is both layer and stimulus specific. For example, responses in L4-barrels were facilitated by "touch", while those in L5a were facilitated in both "touch" and "whisking" conditions and in L2-3 were depressed in both conditions. This is interesting in view of the anatomical and physiological evidence that the various types of whisker-related signals reach the cortex though largely parallel transthalamic pathways: the whisking signals via Po ("paralemniscal"), contact signals via ventrolateral portion of VPM ("extralemniscal") and complex whiskingtouch signals via dorsomedial portion of VPM ("lemniscal"; Yu et al. 2006).

Despite the fact that direct electrical Po stimulation and peripheral sensory receptor stimulation are not directly comparable, we observed different forms of adaptation between different S1BF layers. This pattern is reminiscent of that produced by "whisking" in this area (Derdikman et al. 2006). Adaptation in M1wk was also different from that observed in S1BF. Overall, these differences suggest that the effects of Po synapses on processes such as gain control or optimization of information transmission may be area-specific (Fairhall et al. 2001; Kohn and Whitsel 2002).

In addition, the observation that the effects of Po activation are more pronounced in M1wk neurons is consistent with our previous findings that silencing Po with muscimol drastically reduce evoked potential responses to whisker sensory stimulation in M1wk, while these responses in S1BF remain nearly unchanged (Casas-Torremocha et al. 2017) and unit firing rate increases (Castejon et al. 2016).

\section{Po synapses in S1BF and M1wk are mediated by different glutamate receptor types}

We found that the facilitation elicited by Po stimulation in superficial M1wk layers is partially blocked by the NMDA receptor antagonist D-AP5, but not by the non-selective metabotropic receptor antagonist MCPG. The late component was the most affected, shortening total response duration (Fig. 5c), a finding consistent with NMDA receptors mediating the long-lasting response enhancement to repetitive stimulation (Daw et al. 2006; Remy and Spruston 2007). By contrast, Po-evoked facilitation in S1BF is shortened and partially blocked by both D-AP5 and MCPG (Fig. 4). This supports "in vitro" observations demonstrating the involvement of both receptors in S1BF responses to Po activation (Viaene et al. 2011a).

NMDA receptors participate in the late-lasting component of the S1BF responses to whisker sensory stimulation (Armstrong-James et al. 1993; Banerjee et al. 2009; Barros-Zulaica et al. 2014). Moreover, they have been recently shown to mediate Po-dependent long-term potentiation of S1BF neurons following repetitive whisker stimulation (Gambino et al. 2014; Williams and Holtmaat 2018). In addition, recent studies have begun exploring the role of NMDA receptors in motor cortex (Hasan et al. 2013; Kida and Mitsushima 2018). Our "in vivo" experimental protocol using wild-type animals is not adequate to block the pre- or postsynaptic effect to NMDA on responses to Po stimulation. Thus, we cannot resolve between pre- and postsynaptic NMDA effects. It has to be noted that NMDA presynaptic 
receptors are actually present in layer L4-L2/3 mouse somatosensory cortex synapses (Bender et al. 2006; Brasier and Feldman 2008; Banerjee et al. 2016).

The observed shortening of S1BF responses produced by MCPG is consistent with an involvement of metabotropic receptors in long-lasting response modulation (reviewed in Sherman 2004; Chung and Kim 2017).

Glutamatergic axon terminals have been classified into two broad functional categories according to specific combinations of structural and functional traits (for review see Sherman and Guillery 2013). The first are large terminals with extensive active zones and vesicle pools, in which ionotropic receptor-mediated transmission produces large initial EPSPs, paired-pulse depression and an all-or-none activation profile ("class 1" or "driver" synapses). Terminals of the second category ("class 2" or "modulatory") are smaller and/or have comparably smaller active zones and vesicle pools. They involve both ionotropic and metabotropic receptors, and elicit small EPSPs that show paired-pulse facilitation and graded activation. Distance to the cell soma and/or spatiotemporal convergence may further modify the functional impact of such synapse types (Bruno and Sakmann 2006; Viaene et al. 2011b). Overall, class 1 terminals can "drive" postsynaptic neurons and strongly affect their output, while class 2 terminals are believed to mainly modulate ongoing cellular activity (Chung et al. 2002; Petrof and Sherman 2013).

According to the above criteria, our data can be viewed as an indication that Po synapses effectively "drive" with higher efficacy M1wk neurons, while playing a more "modulatory" role on S1BF neurons. In fact, modulatory-like features of the postsynaptic potentials evoked by Po synapses in S1BF neurons have been observed with intracellular recording methods (Viaene et al. 2011a). A recent "in vitro" study (Mo and Sherman 2019) examined the currents produced by Po synapses in motor cortex neurons, and found relatively large excitatory postsynaptic currents, which showed paired-pulse depression to the optogenetic activation of Po terminals. However, the study did not address the receptor mechanisms involved.

\section{Concluding remarks}

Hierarchically wired architectures may be the cellular substrate for the predictive and error-detection forebrain computations that produce skilled motion and active sensing (Larkum 2013; Ahissar and Assa 2016). In the cerebral cortex, layer-specific patterns of arborization (Crick and Koch 1998; Markov et al. 2014), synaptic bouton size and specific receptor mechanisms (Sherman and Guillery 2013) have all been related to different hierarchical levels of information processing.
In this context, the present study demonstrates that Po signals can simultaneously reach the motor and sensory cortical modules that are involved in active sensing with the vibrissae. Temporal coherence in Po thalamocortical inputs might thus control functional connectivity between the separate cortical areas, which involved in the complex cognitive task that is active whisker exploration in rodents (Saalmann et al. 2012; Nakajima and Halassa 2017; Schmitt et al. 2017). Moreover, our finding that the Po axon terminals target specific layers, have markedly different bouton sizes and involve different receptor mechanisms raises the intriguing possibility that Po signals may have different hierarchical range/operational value in M1wk than in S1BF.

Acknowledgements The authors gratefully acknowledge Ms. Begoña Rodriguez and Ms. Marta Callejo for excellent technical help.

Author contributions DCT, CP, AN and FC designed the study; $\mathrm{CP}$ and MGA performed the neurohistology and stereology analysis; DCT and AN performed the electrophysiology experiments and analysis; JRM and JHRL performed the electron microscopic study. DCT and FC wrote the paper with input from all authors.

Funding This study was supported by European Union's Horizon 2020 (Grant Agreement no. 785907 HBP SGA2) and Ministerio de Economía y Competitividad/Fondo Europeo para el Desarrollo Regional (MINECO/FEDER) Grant BFU2017-88549 to F.C., and MINECO/FEDER Grant BFU2012-36107 to A.N.

\section{Compliance with ethical standards}

Conflict of interest The authors declare that they have no conflict of interest.

Studies involving human participants This article does not contain any studies with human participants performed by any of the authors.

Ethical standards All procedures involving live animals were conducted at the Autónoma de Madrid University under protocols approved by both the University's Ethics Committee and the competent Government agency (Permissions PROEX175/16 and PROEX189/16), in accordance with Spanish law and the European Community Council Directive 2010/63/UE.

Open Access This article is distributed under the terms of the Creative Commons Attribution 4.0 International License (http://creativeco mmons.org/licenses/by/4.0/), which permits unrestricted use, distribution, and reproduction in any medium, provided you give appropriate credit to the original author(s) and the source, provide a link to the Creative Commons license, and indicate if changes were made.

\section{References}

Ahissar E, Assa E (2016) Perception as a closed-loop convergence process. Elife 5:e12830. https://doi.org/10.7554/eLife.12830.001 
Ahissar E, Oram T (2015) Thalamic relay or cortico-thalamic processing? Old question, new answers. Cereb Cortex 25:845-848. https ://doi.org/10.1093/cercor/bht296

Ahissar E, Sosnik R, Haidarliu S (2000) Transformation from temporal to rate coding in a somatosensory thalamocortical pathway. Nature 406:302-306. https://doi.org/10.1038/35018568

Ahissar E, Sosnik R, Bagdasarian K, Haidarliu S (2001) Temporal frequency of whisker movement. II. Laminar organization of cortical representations. J Neurophysiol 86:354-367. https:// doi.org/10.1152/jn.2001.86.1.354

Armstrong-James M, Welker E, Callahan CA (1993) The contribution of NMDA and non-NMDA receptors to fast and slow transmission of sensory information in the rat SI barrel cortex. J Neurosci 13:2149-2160. https://doi.org/10.1523/JNEUROSCI.13-0502149.1993

Audette NJ, Urban-Ciecko J, Matsushita M, Barth AL (2018) POm thalamocortical input drives layer-specific microcircuits in somatosensory cortex. Cereb Cortex 28:1312-1328. https://doi. org/10.1093/cercor/bhx044

Banerjee A, Meredith RM, Rodríguez-Moreno A, Mierau SB, Auberson YP, Paulsen O (2009) Double dissociation of spike timingdependent potentiation and depression by subunit-preferring NMDA receptor antagonists in mouse barrel cortex. Cereb Cortex 19:2959-2969. https://doi.org/10.1093/cercor/bhp067

Banerjee A, Larsen RS, Philpot BD, Paulsen O (2016) Roles of presynaptic NMDA receptors in neurotransmission and plasticity. Trends Neurosci 39:26-39. https://doi.org/10.1016/j. tins.2015.11.001

Barros-Zulaica N, Castejon C, Nuñez A (2014) Frequency-specific response facilitation of supra and infragranular barrel cortical neurons depends on NMDA receptor activation in rats. Neuroscience 281:178-194. https://doi.org/10.1016/j.neuroscien ce.2014.09.057

Bender VA, Bender KJ, Brasier DJ, Feldman DE (2006) Two coincidence detectors for spike timing-dependent plasticity in somatosensory cortex. J Neurosci 26:4166-4177. https://doi. org/10.1523/JNEUROSCI.0176-06.2006

Bermejo PE, Jimenez CE, Torres CV, Avendano C (2003) Quantitative stereological evaluation of the gracile and cuneate nuclei and their projection neurons in the rat. J Comp Neurol 463:419-433. https://doi.org/10.1002/cne.10747

Bickford ME (2016) Thalamic circuit diversity: modulation of the driver/modulator framework. Front Neural Circ 9:86. https:// doi.org/10.3389/fncir.2015.00086

Bopp R, Holler-Rickauer S, Martin KA, Schuhknecht GF (2017) An ultrastructural study of the thalamic input to layer 4 of primary motor and primary somatosensory cortex in the mouse. J Neurosci 37:2435-2448. https://doi.org/10.1523/JNEUR OSCI.2557-16.2017

Bourne JN, Chirillo MA, Harris KM (2013) Presynaptic ultrastructural plasticity along $\mathrm{CA} 3 \rightarrow \mathrm{CA} 1$ axons during long-term potentiation in mature hippocampus. J Comp Neurol 521:3898-3912. https:// doi.org/10.1002/cne.23384

Brasier DJ, Feldman DE (2008) Synapse-specific expression of functional presynaptic NMDA receptors in rat somatosensory cortex. J Neurosci 28:2199-2211. https://doi.org/10.1523/JNEUR OSCI.3915-07.2008

Brecht M, Sakmann B (2002) Dynamic representation of whisker deflection by synaptic potentials in spiny stellate and pyramidal cells in the barrels and septa of layer 4 rat somatosensory cortex. J Physiol 543:49-70. https://doi.org/10.1113/jphys iol.2002.018465

Brecht M, Krauss A, Muhammad S, Sinai-Esfahani L, Bellanca S, Margrie TW (2004) Organization of rat vibrissa motor cortex and adjacent areas according to cytoarchitectonics, microstimulation, and intracellular stimulation of identified cells. J Comp Neurol 479:360-373. https://doi.org/10.1002/cne.20306

Bruno RM, Sakmann B (2006) Cortex is driven by weak but synchronously active thalamocortical synapses. Science 312:1622-1627. https://doi.org/10.1126/science.1124593

Bureau I, von Saint Paul F, Svoboda K (2006) Interdigitated paralemniscal and lemniscal pathways in the mouse barrel cortex. PLoS Biol 4:e382. https://doi.org/10.1371/journal.pbio.0050028 (ErratumPLoS Biol 2007 5:e28)

Casas-Torremocha D, Clascá F, Núñez A (2017) Posterior thalamic nucleus modulation of tactile stimuli processing in rat motor and primary somatosensory cortices. Front Neural Circ 11:69. https://doi.org/10.3389/fncir.2017.00069

Castejon C, Barros-Zulaica N, Nuñez A (2016) Control of somatosensory cortical processing by thalamic posterior medial nucleus: a new role of thalamus in cortical function. PLoS One 11:e0148169. https://doi.org/10.1371/journal.pone.0148169

Chakrabarti S, Zhang M, Alloway KD (2008) MI neuronal responses to peripheral whisker stimulation: relationship to neuronal activity in SI barrels and septa. J Neurophysiol 100:50-63. https://doi.org/10.1152/jn.90327.2008

Chmielowska J, Carvell GE, Simons DJ (1989) Spatial organization of thalamocortical and corticothalamic projection systems in the rat SmI barrel cortex. J Comp Neurol 285:325-338. https ://doi.org/10.1002/cne.902850304

Chung G, Kim SJ (2017) Sustained activity of metabotropic glutamate receptor: homer, arrestin, and beyond. Neural Plast 2017:5125624. https://doi.org/10.1155/2017/5125624

Chung S, Li X, Nelson SB (2002) Short-term depression at thalamocortical synapses contributes to rapid adaptation of cortical sensory responses in vivo. Neuron 34:437-446. https://doi. org/10.1016/S0896-6273(02)00659-1

Clasca F, Porrero C, Galazo M, Rubio-Garrido P, Evangelio M (2016) Anatomy and development of multi-specific thalamocortical axons: implications for cortical dynamics and evolution. In: Rockland KS (ed) Axons and brain architecture. Elsevier, Amsterdam, pp 69-92. https://doi.org/10.1016/B9780-12-801393-9.00004-9

Crick F, Koch C (1998) Constraints on cortical and thalamic projections: the no-strong-loops hypothesis. Nature 391:245-250. https://doi.org/10.1038/34584

Daw MI, Bannister NV, Isaac JT (2006) Rapid, activity-dependent plasticity in timing precision in neonatal barrel cortex. J Neurosci 26:4178-4187. https://doi.org/10.1523/JNEUR OSCI.0150-06.2006

De Chazeron I, Raboisson P, Dallel R (2004) Organization of diencephalic projections from the spinal trigeminal nucleus oralis: an anterograde tracing study in the rat. Neuroscience 127:921928. https://doi.org/10.1016/j.neuroscience.2004.06.005

Derdikman D, Yu C, Haidarliu S, Bagdasarian K, Arieli A, Ahissar E (2006) Layer-specific touch-dependent facilitation and depression in the somatosensory cortex during active whisking. J Neurosci 26:9538-9547. https://doi.org/10.1523/JNEUR OSCI.0918-06.2006

Deschênes M, Veinante P, Zhang ZW (1998) The organization of corticothalamic projections: reciprocity versus parity. Brain Res Brain Res Rev 28:286-308. https://doi.org/10.1016/S0165 $-0173(98) 00017-4$

Drøjdahl N, Nielsen HH, Gardi JE, Wree A, Peterson AC, Nyengaard JR, Eyer J, Finsen B (2010) Axonal plasticity elicits long-term changes in oligodendroglia and myelinated fibers. Glia 58:29-42. https://doi.org/10.1002/glia.20897

Economo MN, Viswanathan S, Tasic B, Bas E, Winnubst J, Menon V, Graybuck LT, Nguyen TN, Smith KA, Yao Z, Wang L, Gerfen CR, Chandrashekar J, Zeng H, Looger LL, Svoboda K 
(2018) Distinct descending motor cortex pathways and their roles in movement. Nature 563:79-84. https://doi.org/10.1038/ s41586-018-0642-9

Ermolyuk YS, Alder FG, Henneberger C, Rusakov DA, Kullmann DM, Volynski KE (2012) Independent regulation of basal neurotransmitter release efficacy by variable $\mathrm{Ca}^{2+}$ influx and bouton size at small central synapses. PLoS Biol 10:e1001396. https://doi.org/10.1371/journal.pbio.1001396

Fairhall AL, Lewen GD, Bialek W, de R Van Steveninck RR (2001) Efficiency and ambiguity in an adaptive neural code. Nature 412:787-792. https://doi.org/10.1038/35090500

Farkas T, Kis Z, Toldi J, Wolff JR (1999) Activation of the primary motor cortex by somatosensory stimulation in adult rats is mediated mainly by associational connections from the somatosensory cortex. Neuroscience 90:353-361. https://doi. org/10.1016/S0306-4522(98)00451-5

Ferezou I, Haiss F, Gentet LJ, Aronoff R, Weber B, Petersen CC (2007) Spatiotemporal dynamics of cortical sensorimotor integration in behaving mice. Neuron 56:907-923. https://doi. org/10.1016/j.neuron.2007.10.007

Frangeul L, Porrero C, Garcia-Amado M, Maimone B, Maniglier M, Clascá F, Jabaudon D (2014) Specific activation of the paralemniscal pathway during nociception. Eur J Neurosci 39:1455-1464. https://doi.org/10.1111/ejn.12524

Franklin KBJ, Paxinos G (2008) The mouse brain in stereotaxic coordinates, Third edn. Elservier, New York

Gambino F, Pagès S, Kehayas V, Baptista D, Tatti R, Carleton A, Holtmaat A (2014) Sensory-evoked LTP driven by dendritic plateau potentials in vivo. Nature 515:116-119. https://doi. org/10.1038/nature13664

Garabedian CE, Jones SR, Merzenich MM, Dale A, Moore CI (2003) Band-pass response properties of rat SI neurons. J Neurophysiol 90:1379-1391. https://doi.org/10.1152/jn.01158.2002

Groh A, Bokor H, Mease RA, Plattner VM, Hangya B, Stroh A, Deschenes M, Acsády L (2014) Convergence of cortical and sensory driver inputs on single thalamocortical cells. Cereb Cortex 24:3167-3179. https://doi.org/10.1093/cercor/bht173

Gundersen HJ, Jensen EB (1987) The efficiency of systematic sampling in stereology and its prediction. J Microsc 147:229-263. https://doi.org/10.1111/j.1365-2818.1987.tb02837.x

Gundersen HJ, Jensen EB, Kiêu K, Nielsen J (1999) The efficiency of systematic sampling in stereology-reconsidered. J Microsc 193:199-211. https://doi.org/10.1046/j.1365-2818.1999.00457 $\mathrm{x}$

Guy N, Chalus M, Dallel R, Voisin DL (2005) Both oral and caudal parts of the spinal trigeminal nucleus project to the somatosensory thalamus in the rat. Eur J Neurosci 21:741-754. https://doi. org/10.1111/j.1460-9568.2005.03918.x

Hasan MT, Hernández-González S, Dogbevia G, Treviño M, Bertocchi I, Gruart A, Delgado-García JM (2013) Role of motor cortex NMDA receptors in learning-dependent synaptic plasticity of behaving mice. Nat Commun 4:2258. https://doi.org/10.1038/ ncomms 3258

Herkenham M (1986) New perspectives on the organization and evolution of nonspecific thalamocortical projections. In: Jones EG, Peters A (eds) Cerebral cortex, vol 5. Plenum, New York, pp 403-445

Holderith N, Lorincz A, Katona G, Rózsa B, Kulik A, Watanabe M, Nusser Z (2012) Release probability of hippocampal glutamatergic terminals scales with the size of the active zone. Nat Neurosci 15:988-997. https://doi.org/10.1038/nn.3137

Hooks BM, Hires SA, Zhang YX, Huber D, Petreanu L, Svoboda K, Shepherd GM (2011) Laminar analysis of excitatory local circuits in vibrissal motor and sensory cortical areas. PLoS Biol 9:e1000572. https://doi.org/10.1371/journal.pbio.1000572
Hooks BM, Mao T, Gutnisky DA, Yamawaki N, Svoboda K, Shepherd GM (2013) Organization of cortical and thalamic input to pyramidal neurons in mouse motor cortex. J Neurosci 33:748760. https://doi.org/10.1523/JNEUROSCI.4338-12.2013

Hooks BM, Lin JY, Guo C, Svoboda K (2015) Dual-channel circuit mapping reveals sensorimotor convergence in the primary motor cortex. J Neurosci 35:4418-4426. https://doi.org/10.1523/ JNEUROSCI.3741-14.2015

Hsu A, Luebke JI, Medalla M (2017) Comparative ultrastructural features of excitatory synapses in the visual and frontal cortices of the adult mouse and monkey. J Comp Neurol 525:2175-2191. https://doi.org/10.1002/cne.24196

Johnson RR, Burkhalter A (1996) Microcircuitry of forward and feedback connections within rat visual cortex. J Comp Neurol 368:383-398. https://doi.org/10.1002/(SICI)1096-9861(19960 506)368:3\%3C383::AID-CNE5\%3E3.0.CO;2-1

Jouhanneau JS, Ferrarese L, Estebanez L, Audette NJ, Brecht M, Barth AL, Poulet JF (2014) Cortical fosGFP expression reveals broad receptive field excitatory neurons targeted by POm. Neuron 84:1065-1078. https://doi.org/10.1016/j.neuro n.2014.10.014

Kida H, Mitsushima D (2018) Mechanisms of motor learning mediated by synaptic plasticity in rat primary motor cortex. Neurosci Res 128:14-18. https://doi.org/10.1016/j.neure s.2017.09.008

Kohn A, Whitsel BL (2002) Sensory cortical dynamics. Behav Brain Res 135:119-126. https://doi.org/10.1016/S0166-4328(02)00139 $-0$

Koralek KA, Jensen KF, Killackey HP (1988) Evidence for two complementary patterns of thalamic input to the rat somatosensory cortex. Brain Res 463:346-351. https://doi.org/10.1016/00068993(88)90408-8

Larkum M (2013) A cellular mechanism for cortical associations: an organizing principle for the cerebral cortex. Trends Neurosci 36:141-151. https://doi.org/10.1016/j.tins.2012.11.006

Li CX, Waters RS (1991) Organization of the mouse motor cortex studied by retrograde tracing and intracortical microstimulation (ICMS) mapping. Can J Neurol Sci 18:28-38. https://doi. org/10.1017/S0317167100031267

Lu SM, Lin RC (1993) Thalamic afferents of the rat barrel cortex: a light- and electron-microscopic study using Phaseolus vulgaris leucoagglutinin as an anterograde tracer. Somatosens Mot Res 10:1-16. https://doi.org/10.3109/08990229309028819

Mao T, Kusefoglu D, Hooks BM, Huber D, Petreanu L, Svoboda K (2011) Long-range neuronal circuits underlying the interaction between sensory and motor cortex. Neuron 72:111-123. https:// doi.org/10.1016/j.neuron.2011.07.029

Maravall M, Petersen RS, Fairhall AL, Arabzadeh E, Diamond ME (2007) Shifts in coding properties and maintenance of information transmission during adaptation in barrel cortex. PLoS Biol 5:e19. https://doi.org/10.1371/journal.pbio.0050019

Markov NT, Vezoli J, Chameau P, Falchier A, Quilodran R, Huissoud C, Lamy C, Misery P, Giroud P, Ullman S, Barone P, Dehay C, Knoblauch K, Kennedy H (2014) Anatomy of hierarchy: feedforward and feedback pathways in macaque visual cortex. J Comp Neurol 522:225-259. https://doi.org/10.1002/cne.23458

Martin-Cortecero J, Nuñez A (2014) Tactile response adaptation to whisker stimulation in the lemniscal somatosensory pathway of rats. Brain Res 1591:27-37. https://doi.org/10.1016/j.brain res.2014.10.002

Matyas F, Sreenivasan V, Marbach F, Wacongne C, Barsy B, Mateo C, Aronoff R, Petersen CC (2010) Motor control by sensory cortex. Science 330:1240-1243. https://doi.org/10.1126/science.11957 97 
Matz J, Gilyan A, Kolar A, McCarvill T, Krueger SR (2010) Rapid structural alterations of the active zone lead to sustained changes in neurotransmitter release. Proc Natl Acad Sci USA 107:88368841. https://doi.org/10.1073/pnas.0906087107

Mease RA, Metz M, Groh A (2016) Cortical sensory responses are enhanced by the higher-order thalamus. Cell Rep 14:208-215. https://doi.org/10.1016/j.celrep.2015.12.026

Meyer HS, Wimmer VC, Hemberger M, Bruno RM, de Kock CP, Frick A, Sakmann B, Helmstaedter M (2010) Cell type-specific thalamic innervation in a column of rat vibrissal cortex. Cereb Cortex 20:2287-2303. https://doi.org/10.1093/cercor/bhq069

Mo C, Sherman SM (2019) A sensorimotor pathway via higher-order thalamus. J Neurosci 39:692-704. https://doi.org/10.1523/ JNEUROSCI.1467-18.2018

Nakajima M, Halassa MM (2017) Thalamic control of functional cortical connectivity. Curr Opin Neurobiol 44:127-131. https://doi. org/10.1016/j.conb.2017.04.001

Noseda R, Jakubowski M, Kainz V, Borsook D, Burstein R (2011) Cortical projections of functionally identified thalamic trigeminovascular neurons: implications for migraine headache and its associated symptoms. J Neurosci 31:14204-14217. https://doi. org/10.1523/JNEUROSCI.3285-11.2011

Ohno S, Kuramoto E, Furuta T, Hioki H, Tanaka YR, Fujiyama F, Sonomura T, Uemura M, Sugiyama K, Kaneko T (2012) A morphological analysis of thalamocortical axon fibers of rat posterior thalamic nuclei: a single neuron tracing study with viral vectors. Cereb Cortex 22:2840-2857. https://doi.org/10.1093/cerco $\mathrm{r} / \mathrm{bhr} 356$

Petreanu L, Mao T, Sternson SM, Svoboda K (2009) The subcellular organization of neocortical excitatory connections. Nature 457:1142-1145. https://doi.org/10.1038/nature07709

Petrof I, Sherman SM (2013) Functional significance of synaptic terminal size in glutamatergic sensory pathways in thalamus and cortex. J Physiol 591:3125-3131. https://doi.org/10.1113/jphys iol.2012.247619

Petrof I, Viaene AN, Sherman SM (2015) Properties of the primary somatosensory cortex projection to the primary motor cortex in the mouse. J Neurophysiol 113:2400-2407. https://doi. org/10.1152/jn.00949.2014

Ranck JB Jr (1975) Which elements are excited in electrical stimulation of mammalian central nervous system: a review. Brain Res 98:417-440. https://doi.org/10.1016/0006-8993(75)90364-9

Remy S, Spruston N (2007) Dendritic spikes induce single-burst longterm potentiation. Proc Natl Acad Sci USA 104:17192-17197. https://doi.org/10.1073/pnas.0707919104

Reynolds ES (1963) The use of lead citrate at high $\mathrm{pH}$ as an electronopaque stain in electron microscopy. J Cell Biol 17:208-212

Rodriguez-Moreno J, Rollenhagen A, Arlandis J, Santuy A, MerchanPérez A, DeFelipe J, Lübke JHR, Clasca F (2018) Quantitative 3D ultrastructure of thalamocortical synapses from the "lemniscal" ventral posteromedial nucleus in mouse barrel cortex. Cereb Cortex 28:3159-3175. https://doi.org/10.1093/cercor/bhx187

Rollenhagen A, Klook K, Sätzler K, Qi G, Anstötz M, Feldmeyer D, Lübke JHR (2015) Structural determinants underlying the high efficacy of synaptic transmission and plasticity at synaptic boutons in layer 4 of the adult rat 'barrel cortex'. Brain Struct Funct 220:3185-3209. https://doi.org/10.1007/s00429-014-0850-5

Rollenhagen A, Ohana O, Sätzler K, Hilgetag CC, Kuhl D, Lübke JHR (2018) Structural properties of synaptic transmission and temporal dynamics at excitatory layer 5B synapses in the adult somatosensory cortex. Front Synaptic Neurosci 10:24. https:// doi.org/10.3389/fnsyn.2018.00024

Rose HJ, Metherate R (2001) Thalamic stimulation largely elicits orthodromic, rather than antidromic, cortical activation in an auditory thalamocortical slice. Neuroscience 106:331-340. https ://doi.org/10.1016/S0306-4522(01)00282-2

Saalmann YB, Pinsk MA, Wang L, Li X, Kastner S (2012) The pulvinar regulates information transmission between cortical areas based on attention demands. Science 337:753-756. https://doi. org/10.1126/science.1223082

Schmitt LI, Wimmer RD, Nakajima M, Happ M, Mofakham S, Halassa MM (2017) Thalamic amplification of cortical connectivity sustains attentional control. Nature 545:219-223. https://doi. org/10.1038/nature22073

Sherman SM (2004) The function of metabotropic glutamate receptors in thalamus and cortex. Neuroscientist 20:136-149. https://doi. org/10.1177/1073858413478490

Sherman SM, Guillery RW (2002) The role of the thalamus in the flow of information to the cortex. Philos Trans R Soc Lond B Biol Sci 357:1695-1708. https://doi.org/10.1098/rstb.2002.1161

Sherman SM, Guillery RW (2013) Functional connections of cortical areas. A new view from the thalamus. MIT Press, Cambrigde. https://doi.org/10.1111/ejn.14069

Shu S, Ju G, Fan LZ (1988) The glucose oxidase-DAB-nickel method in peroxidase histochemistry of the nervous system. Neurosci Lett 85:169-171. https://doi.org/10.1016/0304-3940(88)90346-1

Tennant KA, Adkins DL, Donlan NA, Asay AL, Thomas N, Kleim JA, Jones TA (2011) The organization of the forelimb representation of the C57BL/6 mouse motor cortex as defined by intracortical microstimulation and cytoarchitecture. Cereb Cortex 21:865876. https://doi.org/10.1093/cercor/bhq159

Theyel B, Llano D, Sherman SM (2010) The corticothalamocortical circuit drives higher-order cortex in the mouse. Nat Neurosci 13:84-88. https://doi.org/10.1038/nn.2449

Urbain N, Deschênes M (2007) Motor cortex gates vibrissal responses in a thalamocortical projection pathway. Neuron 56:714-725. https://doi.org/10.1016/j.neuron.2007.10.023

Veinante P, Deschênes M (1999) Single- and multi-whisker channels in the ascending projections from the principal trigeminal nucleus in the rat. J Neurosci 19:5085-5095. https://doi.org/10.1523/ JNEUROSCI.19-12-05085.1999

Viaene AN, Petrof I, Sherman SM (2011a) Properties of the thalamic projection from the posterior medial nucleus to primary and secondary somatosensory cortices in the mouse. Proc Natl Acad Sci USA 108:18156-18161. https://doi.org/10.1073/pnas.11148 28108

Viaene AN, Petrof I, Sherman SM (2011b) Synaptic properties of thalamic input to the subgranular layers of primary somatosensory and auditory cortices in the mouse. J Neurosci 31:12738-12747. https://doi.org/10.1523/JNEUROSCI.1565-11.2011

West MJ, Gundersen HJ (1990) Unbiased stereological estimation of the number of neurons in the human hippocampus. J Comp Neurol 296:1-22. https://doi.org/10.1002/cne.902960102

White EL, Weinfeld E, Lev DL (2004) Quantitative analysis of synaptic distribution along thalamocortical axons in adult mouse barrels. J Comp Neurol 479:56-69. https://doi.org/10.1002/cne.902960102

Williams LE, Holtmaat A (2018) Higher-order thalamocortical inputs gate synaptic long-term potentiation via disinhibition. Neuron. https://doi.org/10.1016/j.neuron.2018.10.049

Wimmer VC, Bruno RM, de Kock CP, Kuner T, Sakmann B (2010) Dimensions of a projection column and architecture of VPM and POm axons in rat vibrissal cortex. Cereb Cortex 20:2265-2276. https://doi.org/10.1093/cercor/bhq068

Wong-Riley MT, Merzenich MM, Leake PA (1979) Changes in the visual system of monocularly sutured or enucleated cats demonstrable with cytochrome oxidase histochemistry. Brain Res 171:11-28. https://doi.org/10.1016/0006-8993(76)90185-2 
Yakoubi R, Rollenhagen A, von Lehe M, Shao Y, Sätzler K, Lübke JHR (2018) Quantitative three-dimensional reconstructions of excitatory synaptic boutons in layer 5 of the adult human temporal lobe neocortex: a fine-scale electron microscopic analysis. Cereb Cortex. https://doi.org/10.1093/cercor/bhy146

Yamawaki N, Borges K, Suter BA, Harris KD, Shepherd GM (2014) A genuine layer 4 in motor cortex with prototypical synaptic circuit connectivity. Elife 19(3):e05422. https://doi.org/10.7554/ eLife.05422
Yu C, Derdikman D, Haidarliu S, Ahissar E (2006) Parallel thalamic pathways for whisking and touch signals in the rat. PLoS Biol 4:e124. https://doi.org/10.1371/journal.pbio.0040124

Publisher's Note Springer Nature remains neutral with regard to jurisdictional claims in published maps and institutional affiliations. 